DINÁMICA ENTRE CANTIDADES Y PRECIOS DE LA CADENA LÁCTEA ARGENTINA: UN ANÁLISIS DE COINTEGRACIÓN ESTRUCTURAL

Dynamics Between Quantities and Prices in Argentine Dairy Chain: A Structural Cointegration Analysis

Jimena Vicentin Masaro 


\section{DINÁMICA ENTRE CANTIDADES Y PRECIOS DE LA CADENA LÁCTEA ARGENTINA: UN ANÁLISIS DE COINTEGRACIÓN ESTRUCTURAL}

\section{Dynamics Between Quantities and Prices in Argentine Dairy Chain: A Structural Cointegration Analysis}

Jimena Vicentin Masaroa

Keywords: SVEC, Prices, Quantities, Dynamics, Argentine dairy chain.

Palabras clave: SVEC, Precios, Cantidades, Dinámica, Cadena láctea argentina.

Clasificación JEL: C32, C53, L66

Received: 11/01/2019

Accepted: 01/10/2019

Published: 10/12/2019

a. Instituto de Economía

Aplicada Litoral (IECAL)

Facultad de Ciencias Económicas,

Universidad del Litoral, Santa Fe, Argentina.

jvicentin@fce.unl.edu.ar

jime.v.masaro@gmail.com

Orcid: 0000-0003-2419-130X

\section{Resumen}

En el presente trabajo se aborda la dinámica entre cantidades y precios, tomando como base una de las actividades económicas con mayor relevancia en el sistema económico argentino, con el fin de analizar las relaciones entre estas variables, claves en el conocimiento del desempeño sectorial. Para tal objetivo, se utilizó un modelo de vectores de corrección de error, para incorporar la simultaneidad existente en la definición de dichas variables endógenas en el contexto multinivel de la cadena productiva; se agregó, además, información exógena sobre características de oferta y demanda de la actividad en sus distintos niveles. A partir de lo anterior, se procedió a realizar un análisis de largo y corto plazo sobre la dinámica encontrada para todos los niveles de la cadena y, como resultado, se observaron relaciones estables de corto y largo plazo, en las que el precio mayorista fue una de las más importantes; además se conoció la dinámica mercado interno-externo y su relación con el nivel primario.

\section{Abstract}

In this work is addresses the dynamics between quantities and prices, at one of the most important economic activities in the Argentine economic system, in order to analyze the relationships between these variables, because they are keys in the knowledge of the sectorial performance. An error correction vector model was used to incorporate the simultaneity existing in the definition of those endogenous variables in a multilevel productive chain context, adding also exogenous information on the supply and demand characteristics of the activity at its different levels was added. To achieve the latter, it was carried out a long and short-term analysis on 
the dynamics found for all levels of the chain. As a result, it was observed stable short and long-term relationships, in which the wholesale price was one of the most important. In addition, it allowed knowing the internal-external market dynamics as its relationship with the primary level.

\section{Introducción}

La cadena láctea es una actividad de gran relevancia en la economía de Argentina. A nivel mundial, se ubica en el decimoprimer puesto de producción láctea, en comparación con la Unión Europea (como bloque) o países como Estados Unidos e India, que poseen niveles de producción muy superiores, a saber, 150, casi go y $75 \%$ mayor al de Argentina, respectivamente (Observatorio de la Cadena Láctea Argentina, OCLA, 2019). Cada una de estas cadenas es una estructura en sí misma con características, formas de organización y relaciones entre agentes que son particulares, y en las que el mercado al que abastecen, la legislación impositiva y normativa determinan tal estructura. A nivel primario, más del $90 \%$ de la producción se concentra en las provincias de Santa Fe, Córdoba, Buenos Aires, Entre Ríos y La Pampa, centro de la cuenca pampeana (Figura 1); la industrialización de la leche cruda también está mayoritariamente ubicada en dicha zona, con industrias tecnológicamente heterogéneas (Depetris Guiguet, Rossini, García Arancibia 83 Vicentin Masaro, 2011). Esta cadena productiva ha sido objeto de diferentes estudios para comprender su funcionamiento, con enfoques diversos, algunos científicos y otros más técnicos (p. ej. Depetris Guiguet, García Arancibia 83 Coronel, 2013; Fernández, 2007: Gallacher, 2000; Lema, 2006; Petrecolla, 2016; Rossini, Vicentin Masaro 8 Depetris Guiguet, 2015). Sobre los primeros, se han tratado de describir los comportamientos económicos de la cadena, pero, en general, de manera segregada por nivel productivo. En particular, en ninguno se ha logrado integrar la dinámica entre cantidades y precios a lo largo de la cadena productiva, incluso cuando este tipo de análisis tiene múltiples ventajas prácticas y teóricas para la toma de decisiones. A todas luces, un estudio de esta naturaleza se visualiza como una herramienta básica para conocer las relaciones multinivel de modo integral, que permite predecir y analizar escenarios que mejoren la toma de decisiones tanto de agentes públicos como privados, en sus distintos roles dentro del sistema productivo.

A nivel internacional, existen estudios científicos sobre la dinámica de sectores productivos agroindustriales generales (p. ej. Cakir $\mathcal{3}$ Balagtas, 2012; Sexton, Sheldon, Mccorriston $\mathcal{E}$ Wang, 2004). De los aplicados a cadenas lácteas, los principales objetivos varían entre análisis de la evolución de cantidades comercializadas $(\mathrm{Yu}, 2012)$; factores relevantes sobre el desempeño de cada nivel ( $\mathrm{p}$. ej. Santeramo, 2014; Zhang \& Li, 2015); determinación de precios (Lajdová \& Bielik, 2013: McGuire, 2012; entre otros); y efectos de políticas (p.ej. Nicholson 83 Stephenson, 2014; Wang, Parsons 8 Zhang, 2010). Dentro de la metodología más implementada están, por un lado, los modelos de ecuaciones estructurales y estructurales dinámicas (SEM y DSEM, por sus siglas en inglés, respectivamente), aunque suelen no tener un desempeño predictivo adecuado (Davidson, Hendry, Srba 8 Yeo, 1978: Mosheim, 2012), debido a sus estrictos supuestos estructurales, que no tienen en cuenta la incorporación de la dimensión temporal de la información (Robledo, 2002). Por otro lado, en varios trabajos se ha sugerido como alternativa los modelos de vectores de corrección de error (VECM, por sus siglas en inglés), que permiten conocer las relaciones entre variables de corto y largo plazo, sin grandes requerimientos de información (tanto de cantidad como de longitud de las series) y que sí tienen un desempeño predictivo adecuado (Arias 83 Torres, 2004; Maddala 83 Kim, 1998). 
Figura 1. Provincias con la mayor participación en la producción lechera argentina

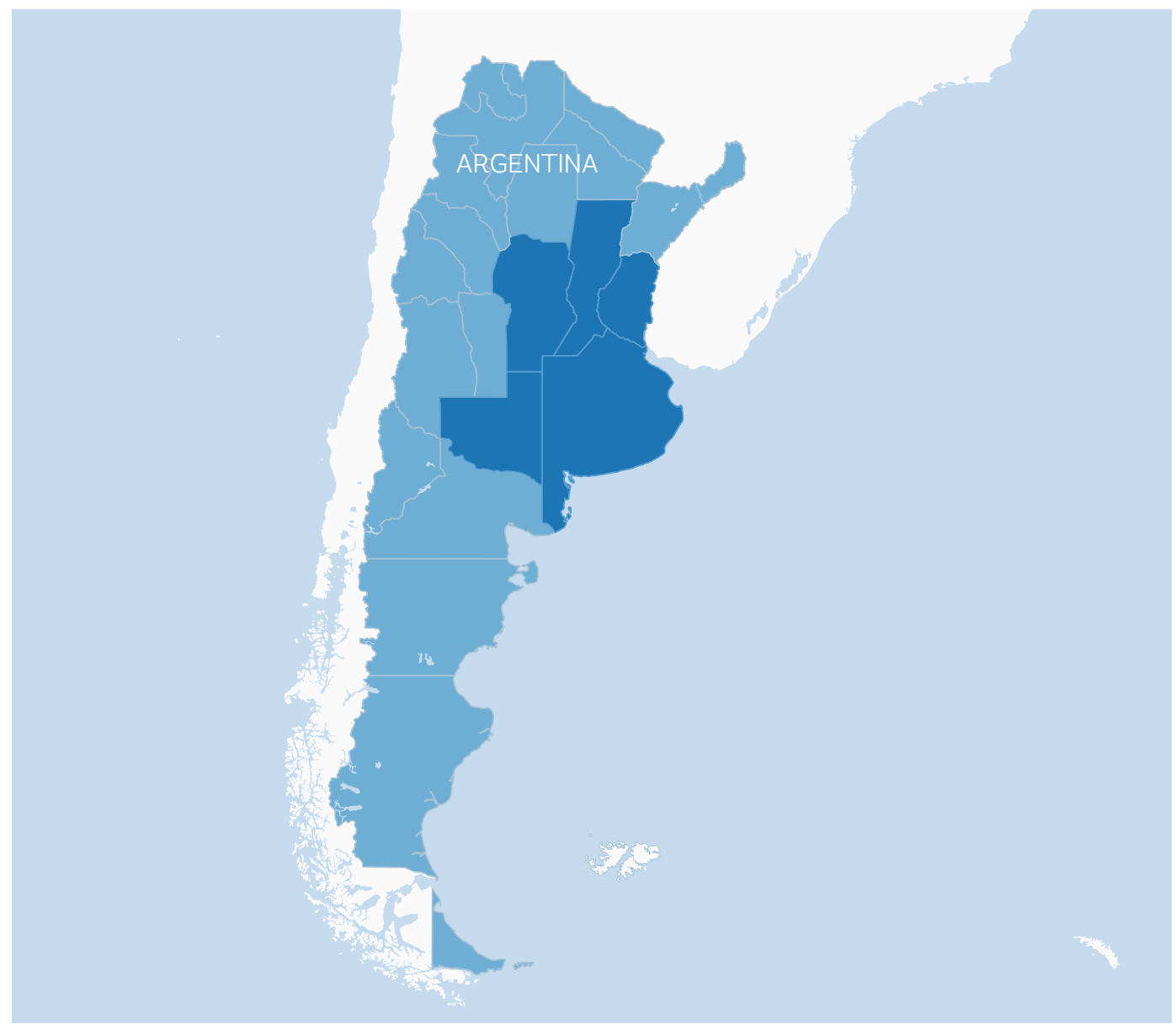

Fuente: elaboración propia.

Este trabajo se presenta entonces como un avance hacia el conocimiento integral y dinámico de una cadena productiva en un país específico. Si bien hay algunos estudios a nivel local, mayoritariamente se sustentan en el análisis segmentado de la cadena, es decir, no se tienen en cuenta los distintos niveles de modo integrado (p. ej. Rossini, Vicentin Masaro, García Arancibia 83 Coronel, 2013: Vicentin Masaro, Depetris Guiguet, García Arancibia 83 Rossini, 2013). En este sentido, el objetivo del presente trabajo fue analizar las relaciones estables de precios y cantidades en el largo plazo, sin perder de vista la integralidad de la cadena específica, para luego estudiar lo que sucede en el corto plazo entre estas variables clave. De esta manera, como aportes originales del presente trabajo se puede mencionar que, en primer lugar, se tuvo en cuenta concomitantemente precios y cantidades, asumiendo endogeneidad entre estas variables y, en segundo lugar, se incorporaron todos los niveles de la cadena y se incluyeron otras variables de oferta y demanda de sus diferentes niveles. Para lograr dicho objetivo, se utilizó el modelo VECM, que tiene en cuenta la simultaneidad en la determinación de variables y la dependencia del tiempo, para conocer las relaciones estables de largo plazo y la evolución en el corto plazo, para luego realizar análisis impulso-respuesta (IRA). Como resultados importantes se encontraron relaciones estables entre cantidades y precios, tanto en el corto como en el largo plazo. Además, las cantidades primarias y las que se ubican en el mercado interno están relacionadas de manera positiva con los precios mayoristas y minoristas, pero no con los precios de la leche cruda; por oposición a las exportaciones, que sí están relacionadas positivamente con estos últimos, pero negativamente con los primeros. 
El artículo se estructura de la siguiente manera: en primer lugar, se realiza la definición del marco económico sobre el que se fundamenta el presente análisis; en segundo lugar, se exponen la estrategia metodológica y los datos utilizados en la aplicación práctica; en tercer lugar, se detallan los resultados y, por último, las conclusiones. Al final del artículo se incluyen como anexos los parámetros estimados adicionales del modelo (véanse tablas A1, A2 y A3).

\section{Modelo económico}

Para efectos de este trabajo, se utilizan los conceptos cadena productiva y teoría de oferta y demanda. En una cadena productiva, los agentes interactúan formando niveles o eslabones interconectados en la evolución del proceso de producción y distribución de los productos (Flórez, Morales Uribe $\mathbb{8}$ Contreras, 2012). Generalmente, se distinguen tres niveles: primario, industrial y de distribución o comercialización; este último puede ser mayorista, exportador o minorista (Guerrero 83 Lilia, 2016). Cada uno es un mercado con fuerzas de demanda y oferta sobre los que, según sus relaciones de corto y largo plazo, se pueden conocer la dinámica de la cadena y los distintos precios que la componen (Cakir 83 Balagtas, 2012; Santeramo, 2014).

En la Figura 2 se resume la estructura de la cadena láctea argentina, sus conexiones entre niveles, donde de la interacción de oferta y demanda se determinan los precios como señales que se transmiten a lo largo de ella. La única demanda no derivada es la de los consumidores finales; las demandas al interior de la cadena devienen del nivel posterior (upstream, véase Figura 2), y los precios que se pagarán también estarán influenciados por la situación y el desempeño del conjunto de los eslabones. Pero, además de los precios, hay otras variables que influyen sobre el desempeño de cada agente y, por consiguiente, de cada nivel. Algunas de estas variables son parte de la estructura de costos y participan del proceso decisorio del agente, como, por ejemplo, los insumos que se utilizan en el proceso de transformación o logística y la tecnología que a corto plazo suele ser exógena. Otras, sin embargo, afectan los niveles productivos y se corresponden con características externas a la decisión del agente; suelen estar asociadas al estado de la naturaleza y entre ellas se pueden mencionar las condiciones políticas, biológicas o los eventos naturales (Ibarra Salazar 8 García Pérez, 2016).

Específicamente, en el presente estudio se parte de las hipótesis de que la industria láctea puede vender en el exterior y al mercado interno (o minorista), pero que no es formadora de precios en el primero y que no hay inventarios en ninguno de los niveles. Este último supuesto es indiscutible para el caso del nivel primario, debido a lo perecedera que es la leche cruda. Sin embargo, para el resto de los niveles existen stocks de corto plazo, aunque la información oficial en este aspecto es inexistente. Como el modelo aplicado no es estructural en las variables, este supuesto no genera problemas en las estimaciones. Se analizan entonces las relaciones de demanda y oferta desde el nivel primario hasta el nivel de distribución minorista. Así, en el nivel primario la cantidad of recida total de leche cruda se fundamenta sobre la decisión óptima de los productores tamberos que, dada la tecnología y el precio de la leche cruda, y de los insumos $\left(w_{l}\right)^{1}$ maximizan su beneficios. Pero su desempeño final depende de cuestiones exógenas como el clima, las políticas sectoriales y las estacionalidades biológicas de la producción, que a priori son inciertas.

1 La simbología en negrita representa matrices. 
Figura 2. Estructura e interrelaciones de la cadena láctea argentina

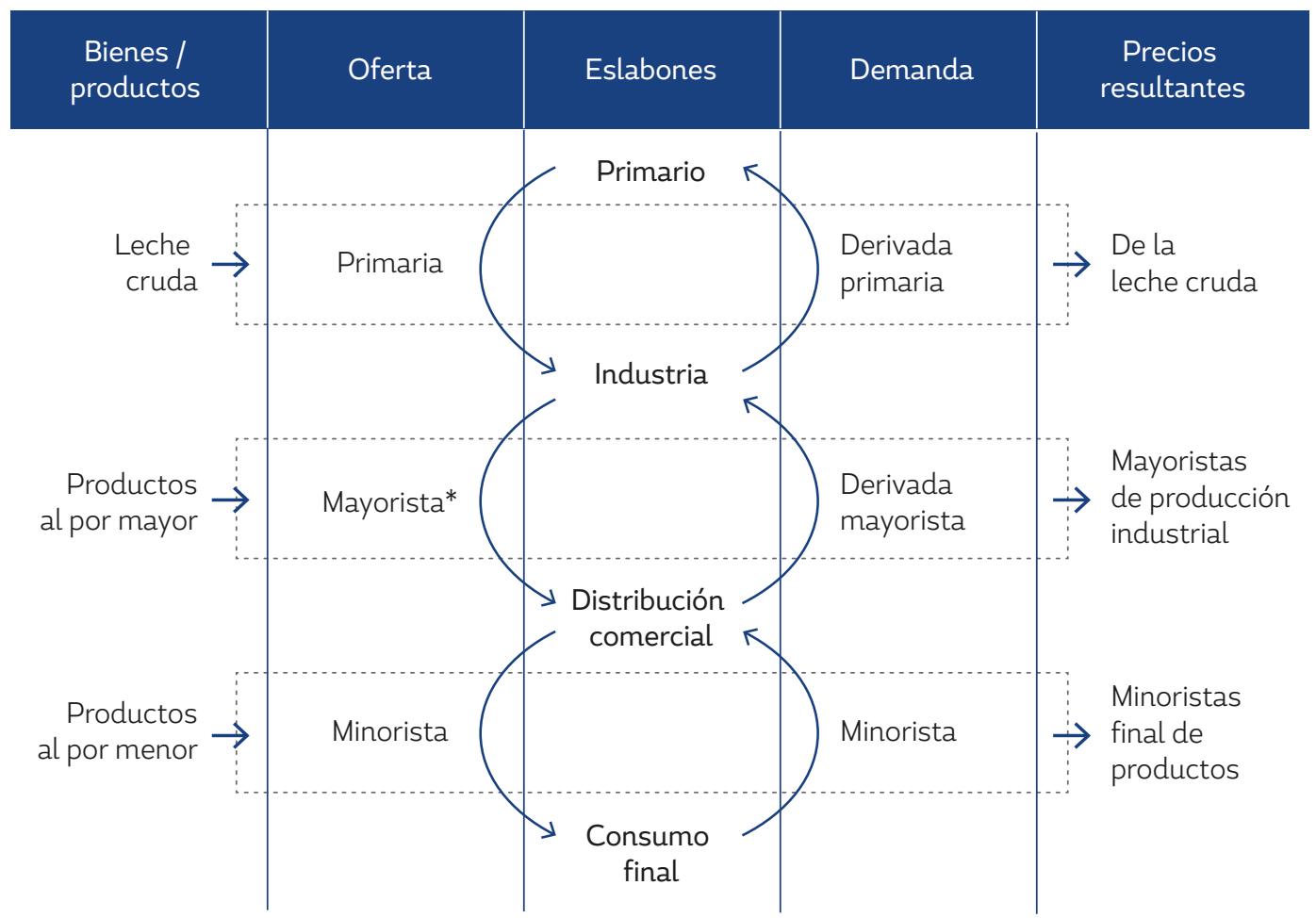

Nota: *se ofrecen productos de exportación, pero la demanda internacional (precios) se considera exógena.

Fuente: elaboración propia.

Esa cantidad de leche cruda (ql) es demandada por los productores industriales (M) que, una vez procesada, venden los productos en el mercado interno y externo ( $m \mathrm{y}^{*}$, respectivamente), por lo que sin perder generalidad, producen dos tipos de bienes: $a_{M}=a_{m}+a_{s}$ Estos industriales deciden cuánto producir de cada uno utilizando factores de producción. Dividiendo estos factores en cantidad de leche cruda y otros $\left(Z_{\mathrm{M}}\right)$, un productor maximiza sus beneficios, dada su función de producción, y de allí obtiene las demandas de estos factores así como la oferta de productos lácteos. Sin perder generalidad, se transforma en litros equivalentes (lte) mediante coeficientes de conversión exógenos (en el corto plazo) para cada conjunto de productos, ${ }^{2}$ entonces la oferta de la producción de la industria en lte es $q_{m}=\Phi_{m}^{T} q_{m}$ y $q_{s}=\Phi^{T}{ }_{v} q_{v}$, respectivamente. ${ }^{3}$

En la comercialización minorista, los agentes demandan al nivel industrial productos que luego distribuyen. Dichos productos no sufren una transformación física, pero se produce un servicio de distribución con costos asociados que se dividen en dos: a) productos lácteos comprados a un precio $P_{M} ; y$ b) otros insumos $\left(z_{m}\right)$ a precios $w_{m}$. De su función de beneficio deciden cuánto demandan de productos lácteos y cuánto of recen en el nivel minorista final a los precios minoristas $\mathrm{p}_{\mathrm{m}}$. La demanda final proviene de la decisión de los consumidores, quienes deciden cuánto consumen de acuerdo con los precios minoristas de los productos lácteos, de otros productos y de su nivel de ingreso. En cuanto a la demanda externa, deviene, en última instancia, también de consumidores finales externos, pero en este trabajo es considerada exógena.

2 Mercado doméstico $(m)$ e internacional $\left({ }^{*}\right)$.

3 Una estrategia similar de agrupamiento es considerada para los precios. 
En equilibrio, las demandas y ofertas de cada nivel se igualan. Por lo tanto, las cantidades son determinadas tanto por variables de demanda como de oferta, siendo

$$
\begin{gathered}
q_{l}=f_{l}\left(p_{l}, \boldsymbol{w}_{l}, \boldsymbol{p}_{*}, \boldsymbol{p}_{M}, \text { clima }, \text { estacionalidades, política, otros }\right) \\
q_{m}=f_{m}\left(p_{*}, p_{M}, w_{M}, w_{m}, p_{m}, \text { otros }\right) \\
q_{*}=f_{*}\left(p_{*}, p_{M}, w_{M}, \text { otros }\right)
\end{gathered}
$$

pero existiendo endogeneidad entre dichas cantidades y precios (excepto el de exportación) e interrelaciones a lo largo de toda la cadena, pues son determinadas simultáneamente.

\section{Metodología}

\subsection{Modelo estadístico}

Para tener en cuenta dichas características, se utiliza un modelo estructural de vectores de corrección de error (SVEC, por sus siglas en inglés). Sea un conjunto de $K$ variables de series de tiempo endógenas $y_{t}=\left(y_{1 t}, \ldots, y_{k t}, \ldots, y_{k t}\right)$ donde $k=1, \ldots, K$, un modelo de Vectores Autorregresivos (VAR) puede capturar las interacciones dinámicas entre dichas variables. Siguiendo a Lütkepohl (2005), un modelo básico de orden p, o $\operatorname{VAR}(p)$, tiene la siguiente forma:

$$
y_{t}=A_{1 t} y_{t-1}+\ldots+A_{p t} y_{t-p}+U_{t}
$$

con matrices de coeficientes $A_{i}$, para $i=(1, \ldots, p)$ de dimensión $(K \mathrm{x} K) ; u_{t}=\left(u_{1 t}, \ldots, u_{k t}\right)^{T}$ es el error inobservable que proviene de un proceso de ruido blanco con media cero e independiente del tiempo. La matriz de covarianzas está definida positiva $\mathrm{E}\left(\mathrm{u}_{\mathrm{t}} \mathrm{u}_{\mathrm{t}}^{\mathrm{T}}\right)=\Sigma_{\mathrm{u}}$, entonces $\mathrm{u}_{\mathrm{t}}:\left(\boldsymbol{0}, \Sigma_{\mathrm{u}}\right)$. Este proceso es estable si $\operatorname{det}\left(I_{k}-A \varsigma-\ldots-A_{p} \varsigma^{p}\right) \neq o$ para $|\varsigma| \leq 1$ es decir, no tiene raíces en el círculo unitario complejo; si las tiene, ${ }^{4}$ entonces algunas (o todas) variables están integradas. Particularmente, cuando el orden de integración es unitario ${ }^{5}$ con tendencia estocástica común, es posible encontrar combinaciones lineales de ellas de forma tal que dicha combinación sea estacionaria, I(o); lo que se denomina cointegración entre variables. La ecuación (4) puede ser reescrita como un modelo de corrección de error (MCE), incluyendo componentes determinísticos y exógenos:

$$
\Delta y_{t}=\Pi_{o}+\Gamma y_{t-1}+\sum_{i=1}^{p} \Pi_{i} \Delta y_{t-i}+\sum_{j=0}^{q} \varphi_{j} X_{t-j}+\Psi D_{t}+u_{t}
$$

donde $\Pi_{o}$ es el vector intercepto de dimensión ( $\left.K \mathrm{x} 1\right)$, $\Pi_{i}$ es la matriz $(K \mathrm{x} K)$ de los coeficientes de corto plazo del i-ésimo rezago definida como: $\Pi_{i}=-\left(A_{i+1}+\ldots,+A_{p}\right)$ para $i=1, \ldots, p-1 ; \Gamma$, la matriz estructural de dimensión $(K x K)$, la cual es $\Gamma=-\left(I_{k}-A_{1}-\ldots-A_{p}\right)$ y, $U_{t}$ es el vector de errores, cuya dimensión es $(K \times 1)$. Las matrices $x_{t}$ y $D_{t}$ corresponden a las de variables exógenas rezagadas en orden $j(p a r a ~ j=1, K, q)$ y a los componentes determinísticos, respectivamente. Entonces, $\Delta y_{t}=y_{t}-y_{t-1}$ no contiene tendencia estocástica y, aunque $y_{t-1}$ son $I(1)$, en su conjunto, $\Gamma y_{t-1}$ debe ser $I(0)$. Entonces, la matriz $\Gamma$ es de rango

4 Es decir, el determinante es cero para.

5 Es decir, son I(1). 
reducido de orden $r$ (es decir $0<r<\mathrm{K})^{\mathrm{t}-1} \mathrm{y}$ se puede descomponer $\Gamma=\alpha \beta^{T}$, donde $\alpha$ es una matriz con los coeficientes de ajustes y $\beta$, los coeficientes de cointegración de largo plazo, con dimensiones $(r \mathrm{x} r) \mathrm{y}(\mathrm{Kx} r)$, respectivamente; y $\beta^{T} y_{t}: I(0)$. En economía estos resultados son muy utilizados porque permiten descomponer las relaciones de las variables entre el corto $\left(\Pi_{j} e n j=1, \ldots, p-1\right)$ y largo plazo $\left(\Gamma y_{t-1}\right)$, y son conocidos como VECM.

Siguiendo a Pfaff (2008), la ecuación (5) puede ser reescrita con $u_{t}=B \varepsilon_{t}, \varepsilon_{t}: N\left(\mathbf{0}, I_{k}\right) \mathrm{y}, \Sigma_{u}=B B^{T}$ para incorporar restricciones estructurales en los errores y definir un SVEC, tal como se propone en el presente trabajo. Una representación de Beveridge-Nelson de medias móviles para $\boldsymbol{y}_{t}^{6}$ es $y_{t}=\Theta \sum_{i=1}^{T} \varepsilon_{i}+\sum_{j=0}^{\infty} \Theta_{j}^{*} \varepsilon_{j-1}+y_{0}^{*}$, donde las variables pueden ser descompuestas en una parte que es $I(1) \mathrm{y}$ otra $I(0)$. El primer término es la tendencia común en el sistema de ecuaciones que dirige el sistema; el término medio es I(0), cuya suma infinita converge a cero, a saber, $\Theta_{j}^{*} \rightarrow 0$ cuando j $\rightarrow \infty$, e $y_{0}^{*}$ son los valores iniciales. La matriz $\Theta$ es de rango $K-r$ donde $r$ es la cantidad de relaciones estables de cointegración y se define como:

$$
\Theta=\beta_{\perp}^{T}\left[\alpha_{\perp}^{T}\left(I_{K}-\sum_{i=1}^{p} \Pi_{i}\right) \beta_{\perp}\right]^{-1} \alpha_{\perp}^{T}
$$

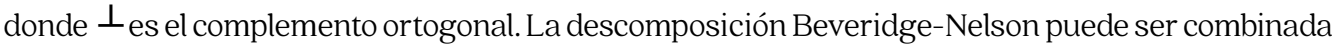
con el término $B \varepsilon_{t}$ del error, entonces las tendencias comunes están dadas por $\Theta B \sum_{t=0}^{\infty} \boldsymbol{\varepsilon}_{t}$, donde los efectos de largo plazo de los shocks estructurales son capturados por la matriz $\Theta B$ y, además, los efectos contemporáneos de los errores estructurales están contenidos en la matriz B. Con $\Theta$ de rango reducido, solo $K-r$ tendencias comunes pueden dirigir el sistema. Siendo r el rango de $\Gamma$, al menos $r$ de los errores estructurales pueden tener un efecto transitorio, lo que significa que r columnas de $\Theta$ son nulas, y esto genera $r(K-r)$ restricciones lineales sobre la matriz $\Theta B$ y sobre el problema en general. La identificación requiere de un total de $\frac{1}{2} K(K-1)$ restricciones lineales, en las que se pueden incorporar las restantes sobre B (efectos contemporáneos). ${ }^{7}$ Esta descomposición de los errores permite incluir restricciones sobre efectos permanentes o contemporáneos en las innovaciones/shocks, incorporando relaciones teóricas a partir de las restricciones de identificación.

\subsection{Variables y fuentes}

3.2.1 Variables exógenas del proceso dinámico

Tal como se menciona en el segundo apartado, los precios y cantidades de los distintos niveles son considerados las variables endógenas. Sin embargo, siguiendo la teoría económica sobre funciones de beneficios (o utilidad) y la disponibilidad de información en el país de aplicación, se incluyen otras variables en la estructura de corto plazo, consideradas exógenas, que influyen en la determinación de las endógenas. Dentro de estas, los shocks tecnológicos, estacionales o climáticos ocurridos en el momento $t$ son incorporados a modo de switch en las estructuras de corto plazo.

Así, las variables exógenas se definen por nivel productivo. En el primario, la cuenca lechera de Argentina se encuentra en una zona que compite con otras producciones, principalmente la soja, por

6 Las cuales siguen un proceso VECM. Ver ecuación (5).

7 En los estudios empíricos, los parámetros de la matriz B son estimados por máxima verosimilitud (ML). 
lo que se utiliza el precio de esta $\left(p_{t}^{\text {soja }}\right)$ como costo de oportunidad y el del maíz ( $p_{t}^{\text {maiz }}$ ), como costo productivo, ya que dicha commodity es componente principal del mix de alimentación de ganado. En el período analizado se produjeron eventos climáticos extremos, es decir sequías e inundaciones en la cuenca lechera, por lo que para capturar este efecto se agrega una variable dicotómica exógena, "Clima ${ }_{t}$, para identificar estos períodos. A su vez, es común la intervención política en este nivel productivo, principalmente con dos objetivos: a) subsidiar en épocas de desastres climáticos o sostener la rentabilidad al productor y b) incentivar la producción en épocas de precios bajos. Así, persiguiendo el segundo objetivo, en algunos meses en el período 2007-2009 se pagaron compensaciones (un monto fijo por litro entregado al mayorista). Para incorporar este efecto, se agregó una variable binaria, "Compt", indicando los períodos cuya política de compensaciones estaba activa. Y, por último, se incluyeron variables estacionales $(E)$ y de tendencia $(t)$ para cambios tecnológicos.

En el nivel industrial, por el lado de la oferta se incluyó a) salarioInd como costo de mano de obra; b) Índice de Precios Internos Mayorista $\left(\operatorname{IPIM}_{t}^{N G}\right)$, para aproximar otros costos de la industria; y c) la Inversión Bruta Interna Fija ( $\left.I B I F_{t}\right)$ como proxy de los niveles de inversión en la industria. Desde la oferta minorista en el nivel de distribución, se agrega el Índice de Precios al Consumidor $\left(I P C_{t}^{N G}\right)$, que aproxima costos del proceso de distribución. De la demanda interna final se tiene en cuenta a) el Estimador Mensual de Actividad Económica $\left(E M A E_{t}\right)$, como indicador del crecimiento económico; b) el nivel de ingreso de los consumidores $\left(\right.$ salario $\left._{t}\right)$, y c) estacionalidad de consumo final $\left(E_{c f}\right)$.

Por último, en la demanda externa exógena se tienen en cuenta solo variables de oferta de las cantidades que se exportan en Argentina. Dentro de estas, las políticas de retenciones llevadas a cabo en el período se incluyen por medio de una variable binaria (Ret) para discriminar los períodos con derechos de exportación activos.

3.2.2 Operacionalización de las variables

En la Tabla 1 se muestran todas las variables que se utilizan en el presente estudio, con sus respectivas fuentes. Para ajustar el modelo, se cuenta con una base de datos mensual que va desde enero del 2000 hasta octubre del 2015, es decir, un total de 190 observaciones.

Tabla 1. Lista de variables y fuentes de la información

\begin{tabular}{l|l|l|l}
\multicolumn{1}{c|}{ Variables } & U. de Medida & ¿Modificada?+ & \multicolumn{1}{c}{ Fuente } \\
\hline Cantidad de leche cruda & mill. lts. & No & Ministerio de Agroindustria (MiniAgri) \\
\hline $\begin{array}{l}\text { Cantidad total mayorista (may) de prod. lácteos } \\
++\end{array}$ & mill. lte. & Sí & MiniAgri \\
\hline $\begin{array}{l}\text { Cantidad minorista (min) final del mix de prod. } \\
\text { lácteos }{ }^{+++}\end{array}$ & mill. lte. & Sí & MiniAgri \\
\hline $\begin{array}{l}\text { Cantidad may. mix de prod. lácteos para el } \\
\text { merc. Interno }\end{array}$ & mill. lte & Sí & MiniAgri \\
\hline Cantidad exportada mix de prod. lácteos & mill. lte & Sí & Aduana, Penta Transaction, MiniAgri \\
\hline Precio de la leche cruda & $\begin{array}{l}\text { Sconstante } \\
\text { (cte)/lt }\end{array}$ & No & $\begin{array}{l}\text { MiniAgri, Instituto Provincial de } \\
\text { Estadisticas } \\
\text { y Censos de Santa Fe (IPEC) }\end{array}$ \\
\hline Precio may. mix de prod. lácteos & Scte/lte & Sí & MiniAgri \\
\hline Precio min. mix de prod. lácteos & Scte/lte & Sí & MiniAgri \\
\hline
\end{tabular}




\begin{tabular}{|c|c|c|c|}
\hline Variables & U. de Medida & ¿Modificada?+ & Fuente \\
\hline Precio de exportación mix de prod. lácteos & S'cte/lte & Sí & $\begin{array}{l}\text { Aduana, Penta Transaction, MiniAgri, } \\
\text { Banco Central de la República Arǵentina } \\
\text { (BCRA) }\end{array}$ \\
\hline Precio del maíz & S cte/tn. & No & Bolsa de Comercio de Rosario (BCR) \\
\hline Precio Interno de la soja & Şcte/tn. & No & BCR \\
\hline $\begin{array}{l}\text { Índice de Precios Interno al por Mayor Nivel } \\
\text { General (NG) }\end{array}$ & Base 2004 & No & $\begin{array}{l}\text { MiniAgri, Dirección Provincial de } \\
\text { Estadísticas } \\
\text { y Censos de San Luis (DPEC) }\end{array}$ \\
\hline Índice de Precios al Productor NG & Base 2004. & No & MiniAgri, DPEC \\
\hline Índice de Precios al Consumidor NG & Base 2004. & No & $\begin{array}{l}\text { Instituto Nacional de Estadísticas y } \\
\text { Censos (INDEC), DPEC }\end{array}$ \\
\hline EMAE & Base 2004. & No & INDEC \\
\hline Índice de Salarios General & Base 2004 & No & INDEC \\
\hline Remuneración a Industria Alimenticia & $\begin{array}{l}\text { Ścte año } \\
2004 .\end{array}$ & Sí & Ministerio de Economía, INDEC \\
\hline Inversión Bruta Interna Fija (IBIF) & $\begin{array}{l}\text { Șcte año } \\
2004 .\end{array}$ & No & INDEC \\
\hline Retenciones a las exportaciones & Binarias & No & Depetris Guiguet et al. (2011; 2013) \\
\hline Compensaciones a prod. primarios & Binarias & No & Depetris Guiguet et al. (2011; 2013) \\
\hline Inundaciones y sequías & Binarias & No & Depetris Guiguet et al. (2011; 2013) \\
\hline $\begin{array}{l}\text { Estacionalidad en producción primaria/ } \\
\text { exportación }\end{array}$ & Binarias & Sí & MiniAgri, Aduana. \\
\hline Estacionalidad en el consumo min. & Binarias & Sí & MiniAgri, Aduana. \\
\hline
\end{tabular}

Notas: + si la variable se modificó, se amplía en el texto.++Es la suma de cantidades vendidas en el mercado minorista y externo. +++ Las cantidades minoristas y mayoristas son idénticas, pero a efectos analíticos se mencionan por separado.

Fuente: elaboración propia.

Debido a que del proceso de industrialización se obtienen una multiplicidad de productos, se realiza una homogeneización a partir de coeficientes de conversión para transformarlos a lte, lo que genera que algunas variables sean modificadas de su versión original. Sea $\Phi_{j} \epsilon R i_{z 0}^{a j}$ vectores de coeficientes de conversión a litros de productos lácteos paraj=\{m," $\}$, la cantidad en lte es $q_{j}=\Phi_{j}^{T} q_{j}$. Estos coeficientes se obtienen del promedio de varias fuentes públicas de información: ${ }^{8}$ Ministerio de Agroindustria (MiniAgri), Oficina Nacional de Control Agropecuario (ONCCA) e Instituto Nacional de Tecnología Industrial (INTI), y son 4,04 lts/kg para crema o nata; 13,4 lts/kg para queso pasta dura; $10 \mathrm{lts} / \mathrm{kg}$ para queso pasta semidura; $8,06 \mathrm{lts} / \mathrm{kg}$ para queso pasta blanda y fresco; 10,3 lts/kg para manteca o mantequilla; 8,32 lts/kg para leche en polvo entera y 12,5 lts/kg para descremada; 4,2 lts $/ \mathrm{kg}$ en dulce de leche y o,92 lts/kg en yogur. La misma estrategia de agrupamiento se usa en los precios, y se obtienen índices de precios mix de productos, cuya ponderación deviene del peso que cada producto tiene en el nivel correspondiente, a saber, mayorista $(M)$, minorista $(m)$ y de exportación (*). 
Tabla 2. Estadísticas descriptivas de las variables

\begin{tabular}{|c|c|c|c|c|}
\hline Variables & Promedio & CV & $\min$ & $\operatorname{Max}$ \\
\hline Consumo minorista (mill. lte) & 692,99 & 10,75 & 520,03 & 914,34 \\
\hline Consumo minorista per cápita (lte/per cápita) & 17,61 & 10,29 & 13,26 & 22,93 \\
\hline Cantidad exportada (mill. lte) & 194,98 & 41,36 & 54,02 & 646,31 \\
\hline Cantidad mayorista ${ }^{+}$(mill. lte) & 887,97 & 12,37 & 639,06 & 1227,69 \\
\hline Producción de leche cruda (mill. lt) & 773,62 & 14,35 & 479,20 & 1018,70 \\
\hline Precio minorista (Ș cte/lte) & 1,13 & 16,30 & 0,82 & 1,61 \\
\hline Precio mayorista (Ș cte/lte) & 0,86 & 17,44 & 0,61 & 1,07 \\
\hline Precio de exportación (Ș cte/lte) & 0,58 & 18,27 & 0,35 & 0,82 \\
\hline Precio de la leche cruda (Ș cte/lt.) & 0,43 & 16,25 & 0,28 & 0,58 \\
\hline Precio de la soja (Ș cte/tn) & 452,71 & 21,15 & 228,30 & 707,26 \\
\hline Precio del maíz (Ș cte/tn.) & 218,48 & 22,58 & 101,57 & 324,85 \\
\hline Índice Salario General & 346,44 & 97,47 & 57,56 & 1370,89 \\
\hline Salario Industria Alimentaria (Ș́ cte/mensual) & 1791,26 & 24,05 & 1010,27 & 3120,33 \\
\hline EMAE & 126,48 & 15,18 & 87,03 & 168,70 \\
\hline IPIM & 243,08 & 89,99 & 43,04 & 901,21 \\
\hline IPC & 243,28 & 88,34 & 58,46 & 893,52 \\
\hline IBIF (mill. Ș cte) & 25758,40 & 39,66 & 7572,94 & 42064,45 \\
\hline
\end{tabular}

Nota: +corresponde a la cantidad informada por MiniAgri.

Fuente: elaboración propia.

Las variables en valores monetarios (definidas en pesos argentinos o ARS) fueron deflactadas mediante índices de cada nivel. Así, en los precios minoristas y sueldos se utiliza el IPC; en el nivel mayorista y de exportación, el IPIM; y en el nivel primario se deflactan por medio del Índice de Precios al Productor (IPP). En todos los casos se toma como año base el 2004. Cabe destacar que debido a los altos niveles inflacionarios de Argentina resulta importante deflactar las unidades monetarias.

Por otro lado, las variables estacionales surgen de un análisis de estacionalidad observada de la producción de cada nivel y del consumo. Como resultado, la producción primaria y cantidades exportadas tienen valores superiores a su media general entre agosto-enero; a nivel mayorista no se observa un patrón de estacionalidad y en el nivel final se observa estacionalidad en los meses de marzo y diciembre. ${ }^{9}$ Estos patrones de estacionalidades son incorporados en el nivel primario y exportador con la inclusión de variables indicadoras de trimestres: E1 para el trimestre de noviembre a enero, E2 de febrero a abril, E3 de mayo a junio y E4 de agosto a octubre; y de la estacionalidad en el nivel final con la inclusión de una variable binaria Ecf, igual a uno en marzo y diciembre, y cero en el resto del año.

En la Tabla 2 se muestran estadísticos descriptivos de las variables utilizadas (excepto las binarias), para el período 2000-2015. El promedio de las cantidades destinadas al mercado interno son superiores a las exportadas, y estas, más variables. Con respecto a los precios, los minoristas son en promedio $30 \%$ superiores a los mayoristas, y estos dos veces mayores a los precios promedio de la leche cruda. 
3.3 Implementación del modelo dinámico.

Se estima un SVEC incluyendo variables exógenas y determinísticas para conocer relaciones de cor to y largo plazo entre las variables endógenas. Estas variables junto con las monetarias son consideradas en logaritmo. En primer lugar, se realizan pruebas de estacionariedad de las variables, ${ }^{10}$ por medio de las pruebas Dickey-Fuller aumentada (ADF) y Phillip-Perron (PP); la selección del número óptimo de rezagos incluidos en el VAR se hace a partir del criterio de información de Akaike (AIC), siguiendo a」Johansen (1988).

El modelo con variables exógenas o determinísticas se estima en dos etapas: en la primera, se obtiene la matriz de cointegración mediante la metodología de Johansen (Lütkepohl \& Krätzig, 2005) y, en la segunda, se toma $\beta y_{t-1}$ de la primera etapa para estimar los parámetros de corto plazo y de las variables exógenas/determinísticas. Dado que no se imponen restricciones sobre la matriz de variables, la metodología de estimación es de mínimos cuadrados ordinarios (OLS). Por último, se analizan los residuos obtenidos para verificar normalidad, homocedasticidad y no correlación serial.

Las restricciones del SVEC se realizan sobre los errores y, con ello, se realiza el IRA, estudiando la evolución de las variables endógenas ante cambios "no predecibles" ${ }^{\text {"1 }}$ en los errores del modelo. Para la identificación se imponen las siguientes restricciones en los errores: a) la información de los vectores de cointegración en $\Theta B$ y (b) en la matriz de efectos contemporáneos $(B)$ se supone que un shock no previsto: 1) en la cantidad de leche cruda no genera impacto inmediato en los precios minoristas; 2) en la cantidad exportada no produce cambios inmediatos en las cantidades de leche cruda ni en los precios mayoristas; 3) en el precio de la leche cruda no afecta inmediatamente las cantidades exportadas, y 4) en los precios minoristas no genera efectos contemporáneos sobre las cantidades exportadas ni en el precio de la leche cruda. Estas restricciones son impuestas a priori, fundamentadas sobre la dinámica de la estructura productiva de esta cadena.

\section{Resultados}

La prueba ADF indica que todas las variables son I(1) al nivel de $5 \%$, excepto la cantidad exportada y el precio minorista que lo son al nivel $10 \%$. Sin embargo, en la prueba de PP todas son I(1) al nivel del $5 \%$ permitiendo la utilización del SVEC. El modelo completo está compuesto por seis variables endógenas, nueve exógenas y ocho determinísticas. En el vector de diferencias se seleccionan tres rezagos, según el criterio de AIC. En la evaluación pos-estimación se observa que los residuos no tienen autocorrelación serial, heteroscedasticidad y son estacionarios, aunque no se constata normalidad de los residuos. Dado que se tiene una serie relativamente corta, se procede a utilizar la prueba ajustada para muestras pequeñas, y se verifica así normalidad en sus residuos.

Mediante la prueba de cointegración de Johansen, se encuentran al menos tres vectores de cointegración, ${ }^{12}$ por lo que se normalizan estas relaciones en función de las cantidades en cada nivel, es decir, la cantidad de leche cruda $\left(\ln \left(q_{l}\right)=\ln (q l)\right)$, la cantidad al mercado interno $\left(\ln \left(q_{m}\right)=\ln (q m)\right)$ y la cantidad exportada $\left(\ln \left(q_{*}\right)=\ln \left(q^{*}\right)\right)$; esto explica las relaciones en torno a los precios de la leche $\left(\ln \left(p_{l}\right)=\ln (p l)\right)$, mayoristas $\left(\ln \left(p_{M}\right)=\ln (p M)\right)$, minoristas $\left(\ln \left(p_{m}\right)=\ln (p m)\right)$, y el precio de exportación $\left(\ln \left(p_{*}\right)=\ln \left(p^{*}\right)\right)$ es exógeno.

\footnotetext{
10 Aunque interese principalmente el orden de integración de las variables endógenas, también se realiza para las exógenas.

11 "No predecibles" significa que no pueden ser conocidos a través del pasado por medio de los parámetros.

12 Para un $\mathrm{HO} \leq 3$ el estadístico de traza es 26,09, cuyo valor de referencia es 35,07 para un nivel de $5 \%$.
} 
4.1 Relaciones estables de largo y ajuste de corto plazo en la cadena.

En la Tabla 3 se muestran los parámetros estimados de los tres vectores de cointegración. ${ }^{13}$ En el primero (fila a), la normalización está hecha sobre las cantidades de leche cruda. Este resultado muestra que, en el largo plazo, la cantidad producida de leche cruda está relacionada de manera positiva con todos los precios de la cadena. Sin embargo, se destaca el hecho de que el coeficiente correspondiente al precio de la leche no sea significativo. Esto podría deberse a la relación heterogénea que hubo entre precios de la leche cruda y las cantidades a lo largo del período bajo estudio: Argentina tuvo una gran caída de los precios reales de la leche cruda a partir del 2010, con crisis y conflictos políticos en el nivel primario de los que aún no supera y que, más aun, han sido recurrentes en los últimos quince años. Se cree que tales condiciones resultan en una falta de relación estable en el largo plazo entre estas variables.

Un aumento de precios en algún eslabón posterior de la cadena implicaría mejores condiciones de oferta primaria, que, por expectativas de mayores ganancias futuras, favorecen a las cantidades efectivas. La mayor relación se da con los precios mayoristas; cuando estos aumentan un $10 \%$, las cantidades de leche cruda se incrementan en un 5,3\%. Esto podría deberse a que los incrementos de precios de los bienes que se producen incentivan la oferta en este nivel y, por consiguiente, se incrementa la demanda de factores. Sucede lo mismo con los precios minoristas, pero en menor proporción.

Tabla 3. Vectores de cointegración (ec·)

\begin{tabular}{|c|c|c|c|c|c|c|}
\hline $\begin{array}{l}e c 1_{t-1} \\
1 \\
\text { (a) }\end{array}$ & $\ln \left(q_{1}\right)_{t-1}$ & $=$ & $\begin{array}{l}0,104 \ln \left(p_{1}\right)_{t-1} \\
{[0,367]}\end{array}$ & $\begin{array}{l}+0,53 \ln \left(p_{M}\right)_{t-1}^{*} \\
{[0,000]}\end{array}$ & $\begin{array}{l}+0,178 \ln \left(p_{m}\right)_{t-1}^{* *} \\
{[0,015]}\end{array}$ & $+6,442 *$ \\
\hline$e c 2_{t-1}$ & $\ln _{1}\left(q_{m}\right)_{t-1}$ & $=$ & $-0,158 \ln \left(p_{1}\right)_{t-1}$ & $+0,468 \ln \left(p_{M}\right)_{t-1}^{*}$ & $+0,271 \ln \left(p_{m}\right)_{t-1}^{* *}$ & $+6.296^{*}$ \\
\hline$\stackrel{1}{(b)}$ & & & {$[0,431]$} & {$[0,005]$} & {$[0,029]$} & {$[0,000]$} \\
\hline$e c 3_{t-1}$ & $\ln \left(q_{*}\right)_{t-1}$ & $=$ & $0,335 \ln \left(p_{1}\right)_{t-1}^{* *}$ & $-1,201 \ln \left(p_{M}\right)_{t-1}^{*}$ & $-0,888 \ln \left(p_{m}\right)_{t-1}^{* *}$ & $+5,209^{*}$ \\
\hline$\left(\begin{array}{l}1 \\
\text { (c) }\end{array}\right.$ & & & {$[0,042]$} & {$[0,001]$} & {$[0,047]$} & {$[0,000]$} \\
\hline
\end{tabular}

Por otro lado, los coeficientes de reacción de corto plazo (Tabla 4) muestran cómo se modifica cada variable en el proceso hacia el equilibrio estable encontrado en cada vector de cointegración, y su significancia, y que los errores de equilibrio pasado son importantes para obtener nuevamente el equilibrio de largo plazo. Así, los coeficientes de reacción del primer vector (Tabla 4, fila a) muestran que, ante un desequilibrio en el largo plazo, la cantidad de leche cruda y los precios mayoristas son los que se modifican para volver al equilibrio; así, la primera se acomoda con mayor velocidad al desajuste, y los precios mayoristas, con menor velocidad. Esto último deviene del tamaño de los coeficientes de carga.

13 Por conveniencia en la exposición, el resto de los parámetros del SVEC se muestran como anexos, excepto la matriz de carga. 
Tabla 4. Coeficientes de carga o efectos de corto plazo

\begin{tabular}{|c|c|c|c|c|c|c|}
\hline & $\Delta \ln \left(q_{1}\right)$ & $\Delta \ln \left(q_{m}\right)$ & $\Delta \ln \left(q_{*}\right)$ & $\Delta \ln \left(p_{1}\right)$ & $\left.\Delta \ln \left(p_{M}\right)\right)$ & $\Delta \ln \left(p_{m}\right)$ \\
\hline$e c 1_{t-1}(a)$ & $\begin{array}{l}-0,5^{*} \\
{[0,000]}\end{array}$ & $\begin{array}{l}-0,046 \\
{[0,730]}\end{array}$ & $\begin{array}{l}0,017 \\
{[0,954]}\end{array}$ & $\begin{array}{l}-0,008 \\
{[0,790]}\end{array}$ & $\begin{array}{l}-0,047^{* *} \\
{[0,026]}\end{array}$ & $\begin{array}{l}-0,019 \\
{[0,590]}\end{array}$ \\
\hline$e c 2_{t-1}(b)$ & $\begin{array}{l}0,161^{*} \\
{[0,000]}\end{array}$ & $\begin{array}{l}-0,402^{*} \\
{[0,000]}\end{array}$ & $\begin{array}{l}-0,116 \\
{[0,561]}\end{array}$ & $\begin{array}{l}0,009 \\
{[0,679]}\end{array}$ & $\begin{array}{l}0,02 \\
{[0,166]}\end{array}$ & $\begin{array}{l}0,047^{* *} \\
{[0,056]}\end{array}$ \\
\hline$e c 3_{t-1}(c)$ & $\begin{array}{l}-0,01 \\
{[0,580]}\end{array}$ & $\begin{array}{l}-0,019 \\
{[0,612]}\end{array}$ & $\begin{array}{l}-0,373^{*} \\
{[0,000]}\end{array}$ & $\begin{array}{l}0,025^{*} \\
{[0,004]}\end{array}$ & $\begin{array}{l}0,012^{* *} \\
{[0,051]}\end{array}$ & $\begin{array}{l}0,018^{+} \\
{[0,085]}\end{array}$ \\
\hline
\end{tabular}

Notas: la información entre corchetes son los p-valores. $\Delta$ siǵnifica variación. Siǵnificativo al nivel *1\%, ${ }^{* *} 5 \%,{ }^{+} 10 \%$.

Fuente: elaboración propia.

El segundo vector de cointegración normalizado sobre la cantidad de lácteos destinados al mercado interno (Tabla 3, fila b) muestra una relación estable positiva con respecto al precio mayorista y minorista, pero negativa respecto a los precios de la leche cruda, aunque esta última no es significativa. Los productos lácteos son muy importantes en el consumo local nacional, representan aproximadamente el $85 \%$ del total de leche cruda producida (Depetris Guiguet et al., 2011). Este podría ser el origen de la no significatividad de los precios de la leche cruda en dicha relación de largo plazo, pues se siguen vendiendo aun cuando los costos de producción se incrementan.

De las relaciones significativas, cuando los precios del mix mayorista (o minorista) aumentan un $10 \%$, la cantidad de lácteos en el mercado interno se incrementan en 4,7\% (2,7\%). Así, las cantidades están más asociadas a los precios mayoristas que a los minoristas. En el corto plazo, un desequilibrio es ajustado por las cantidades destinadas al mercado interno, así como de leche cruda y los precios minoristas, siendo las primeras las que reaccionan con mayor velocidad; en casi dos meses se reestablece el equilibrio de largo plazo (Tabla 4, fila b).

Por último, el tercer vector de cointegración normalizado en las cantidades del mix exportado (Tabla 3, fila c) muestra una asociación positiva con los precios de la leche cruda, pero negativa con los mayoristas y minoristas, donde todos los parámetros estimados son estadísticamente significativos. Cuando el precio de la leche cruda se incrementa en $10 \%$, las cantidades exportadas lo hacen en 3,4\%, aproximadamente. Se cree que ello ocurre debido a la transmisión de precios que existe desde el mercado internacional al nivel primario, que ha sido corroborado en varios trabajos (Gustavo Rossini et al., 2013: Vicentin Masaro et al., 2013; Vicentin Masaro, Depetris Guiguet, Rossini 83 Coronel, 2012). Precios internacionales más altos no solo incentivan las exportaciones, sino que también influyen en los precios de la leche cruda y, con ello, en un aumento de la producción primaria, por lo que adquieren un rol de referencia en el mercado local primario.

La asociación negativa que se observa entre las cantidades exportadas y los precios mayoristas (y minoristas) indica que el mercado externo es un destino alternativo para la producción, que compite con el interno. Entre ambos precios, el mayorista es de mayor asociación inversa con las exportaciones (en lte). A corto plazo, el desajuste en el equilibrio estable es corregido por todos los precios y por las cantidades exportadas, siendo esta última la que se ajusta con mayor velocidad, a saber, el $37 \%$ el primer mes, mientras que los precios lo hacen de manera más gradual. 
Para una mejor interpretación de los resultados del IRA, se debe tener en cuenta que cada variable reacciona de manera directa a un shock sobre sí misma. Además, debido a las restricciones impuestas por la normalización de cointegración, los shocks sobre cantidades son siempre temporarios. En la Figura 3 se muestra la respuesta de cada una de las variables de cantidades a shocks en el resto de variables endógenas.

Por un lado, la dinámica de las cantidades de leche cruda (en logaritmo) ante distintos shocks en las variables endógenas se muestran en las subfiguras 3.a y 3.b. Cambios imprevistos en las cantidades de productos lácteos destinadas al mercado interno $(\mathrm{m})$ y externo (*) tienen efectos temporarios directos sobre la cantidad de leche cruda, con una reacción más inmediata a la primera variable. Esto puede deberse a que los bienes exportados no son perecederos y, por tanto, el shock puede ser amortiguado inmediatamente. Variaciones no anticipadas en los precios, en cambio, tienen efectos permanentes sobre la cantidad de leche cruda. Si estos shocks implican aumentos de precios en cualquier nivel, las cantidades reaccionan positivamente, pero la repuesta inmediata es mayor a shocks en el precio minorista.

En las subfiguras 3.c y 3.d se muestra la reacción de las cantidades de productos lácteos destinadas al mercado interno ante shocks en el resto de variables endógenas. Con efectos temporarios, estas cantidades responden directamente a un shock en la cantidad de leche cruda e inversamente ante uno en las exportaciones. Se considera que lo primero se debe a que mayores volúmenes no anticipados de leche cruda implican más productos lácteos, tanto para el mercado exportador (Figura 3.e) como también para el interno (Figura 3.c). La reacción indirecta de las cantidades de productos lácteos al mercado interno ante un shock en las cantidades exportadas puede deberse a que un aumento exógeno en las exportaciones genera menor disponibilidad relativa, pero transitoria, para el mercado interno. Por otro lado, un shock en el precio de la leche cruda genera un efecto permanente indirecto sobre la cantidad de productos lácteos destinados al mercado interno, con rezago de un mes en la transmisión, mientras que shocks en el precio mayorista o minorista producen efectos permanentes y directos sobre dicha cantidad hacia el mercado interno. Estos resultados podrían deberse a que los aumentos de estos últimos precios implican una mejora relativa de dicho mercado.

La reacción de las cantidades exportadas ante cambios imprevistos en las variables se muestra en las Figuras 3.e y 3.f. Se puede ver que el efecto inmediato de estas cantidades de lácteos exportadas es contrario a cualquier shock de precios, es decir, de la leche cruda, minorista o mayorista. Solo un cambio inesperado en el precio de la leche cruda es el que está asociado a un cambio directo y permanente en las cantidades de lácteos para exportación; los otros dos precios también son permanentes pero indirectos. Estos resultados pueden deberse a que, con precios superiores (mayoristas o minoristas), el mercado interno se vuelve más atractivo.

En un sentido dinámico e integrador, se esperaría una reacción directa de los precios de la leche cruda ante un shock en las cantidades exportadas de productos lácteos (al menos con rezago) debido a la presión que se genera sobre la demanda de leche cruda para su procesamiento; sin embargo, esto no se observa en los resultados (Figura 4.a). Lo anterior puede deberse a que un cambio inesperado de los precios sea cubierto por stocks, no ejerciendo presiones mayores sobre el desempeño interno. 
Figura 3. Respuesta de las cantidades de productos lácteos de los diferentes niveles en la cadena láctea a shocks en variables endógenas

Respuesta de la cantidad de leche cruda
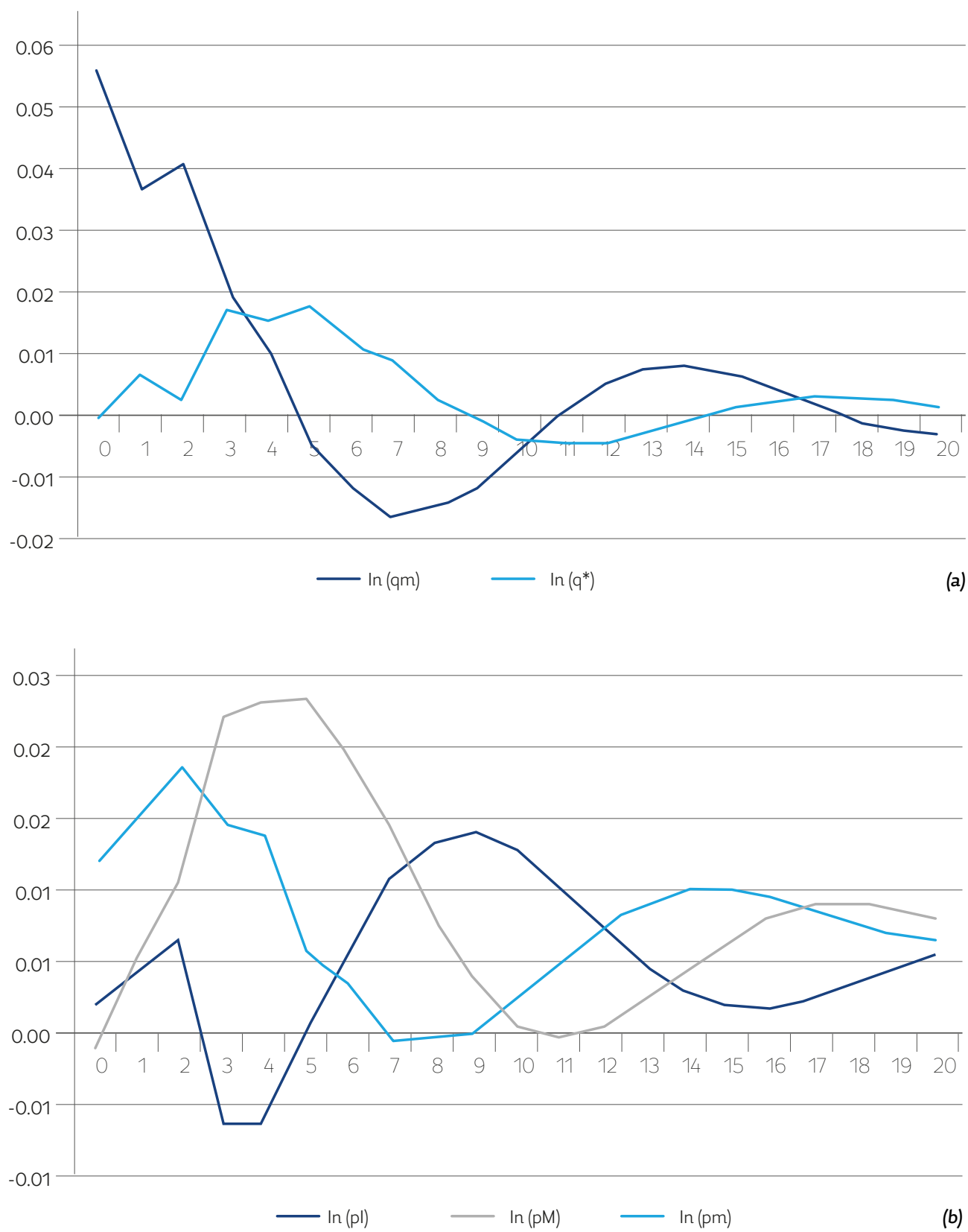
Respuesta de la Cantidad de Lácteos al Mercado Interno (lte)
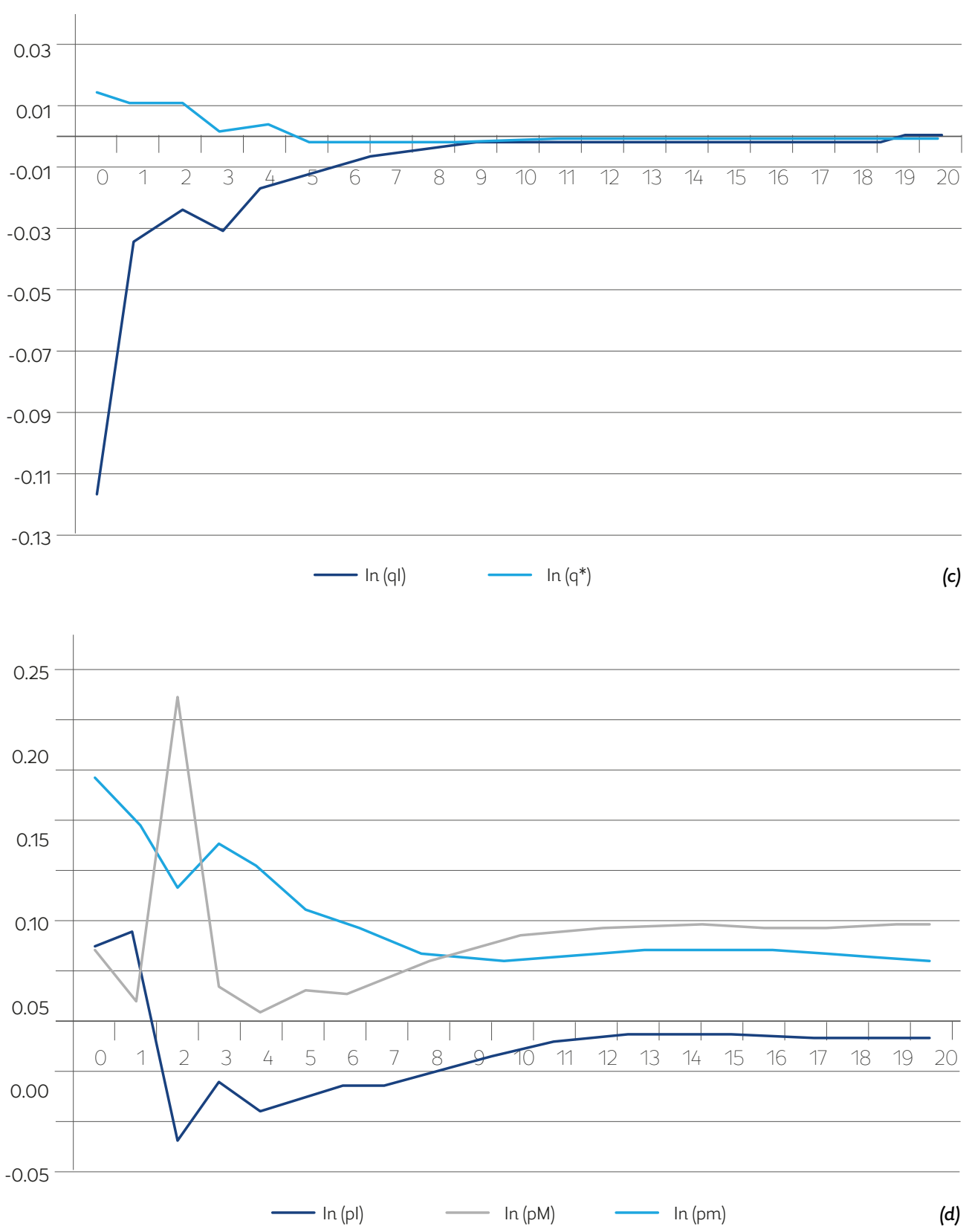
Respuesta de la cantidad de lácteos a exportación (ltc)
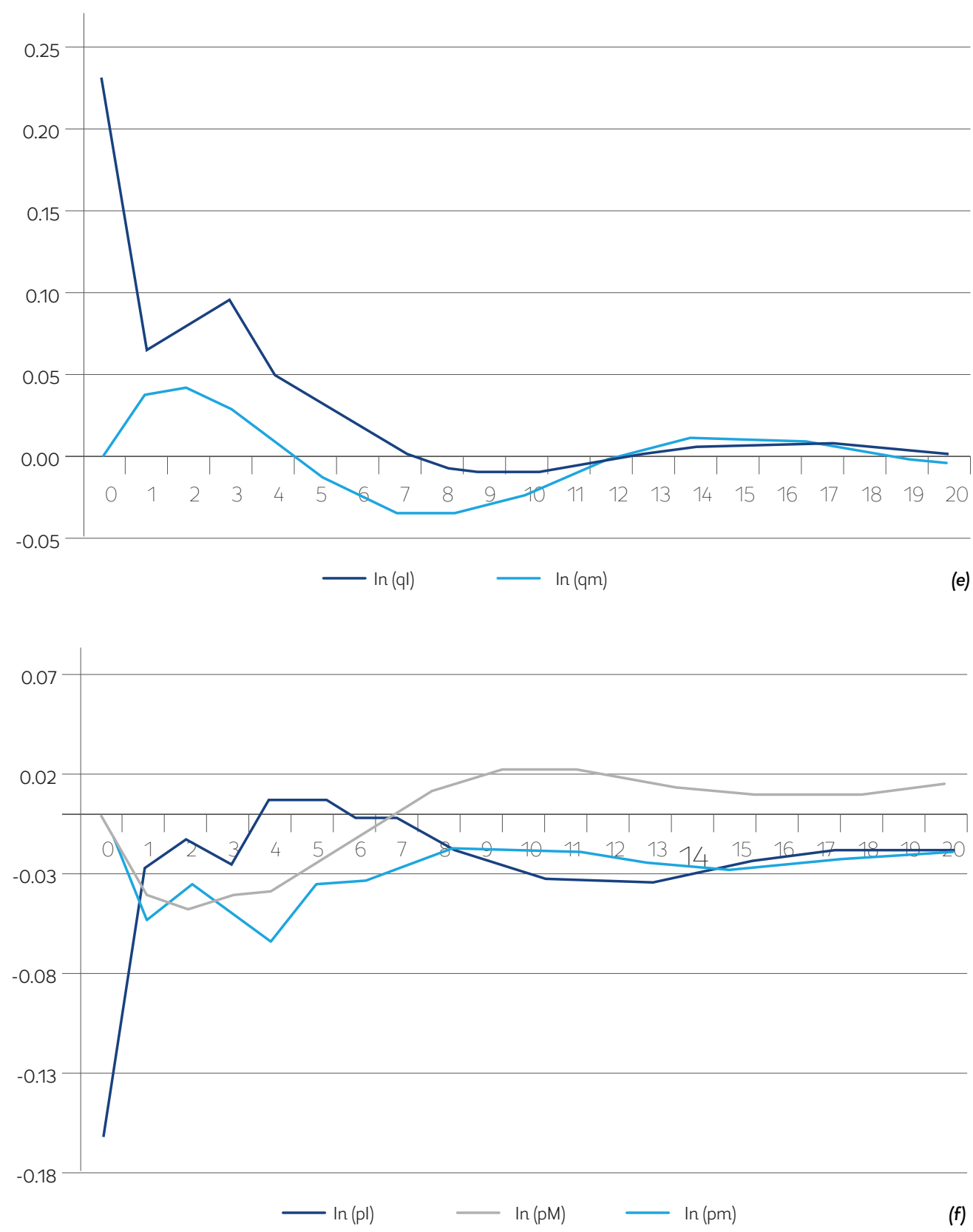

Fuente: elaboración propia.

exógenas en la cantidad exportada, lo que muestra los efectos del trade off en los mercados alternativos de colocación de productos. La variación imprevista de los precios de la leche cruda y minorista, por el contrario, tiene efectos permanentes sobre los precios mayoristas y son incorporados casi de inmediato, siendo indirectos y directos, respectivamente. 
Figura 4. Respuesta de los precios de los distintos niveles en la cadena láctea Respuesta del precio de leche cruda
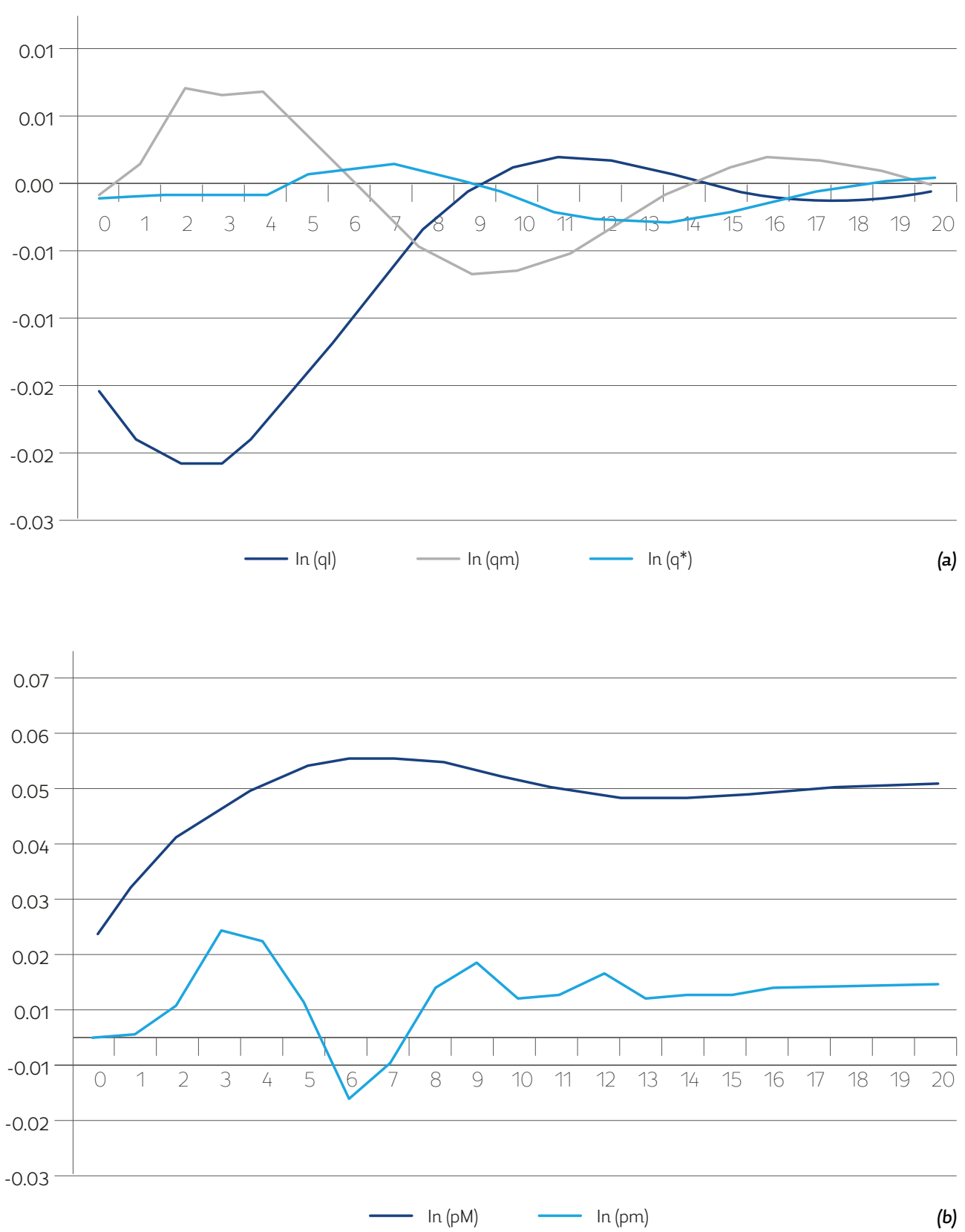
Respuesta del precio mayorista
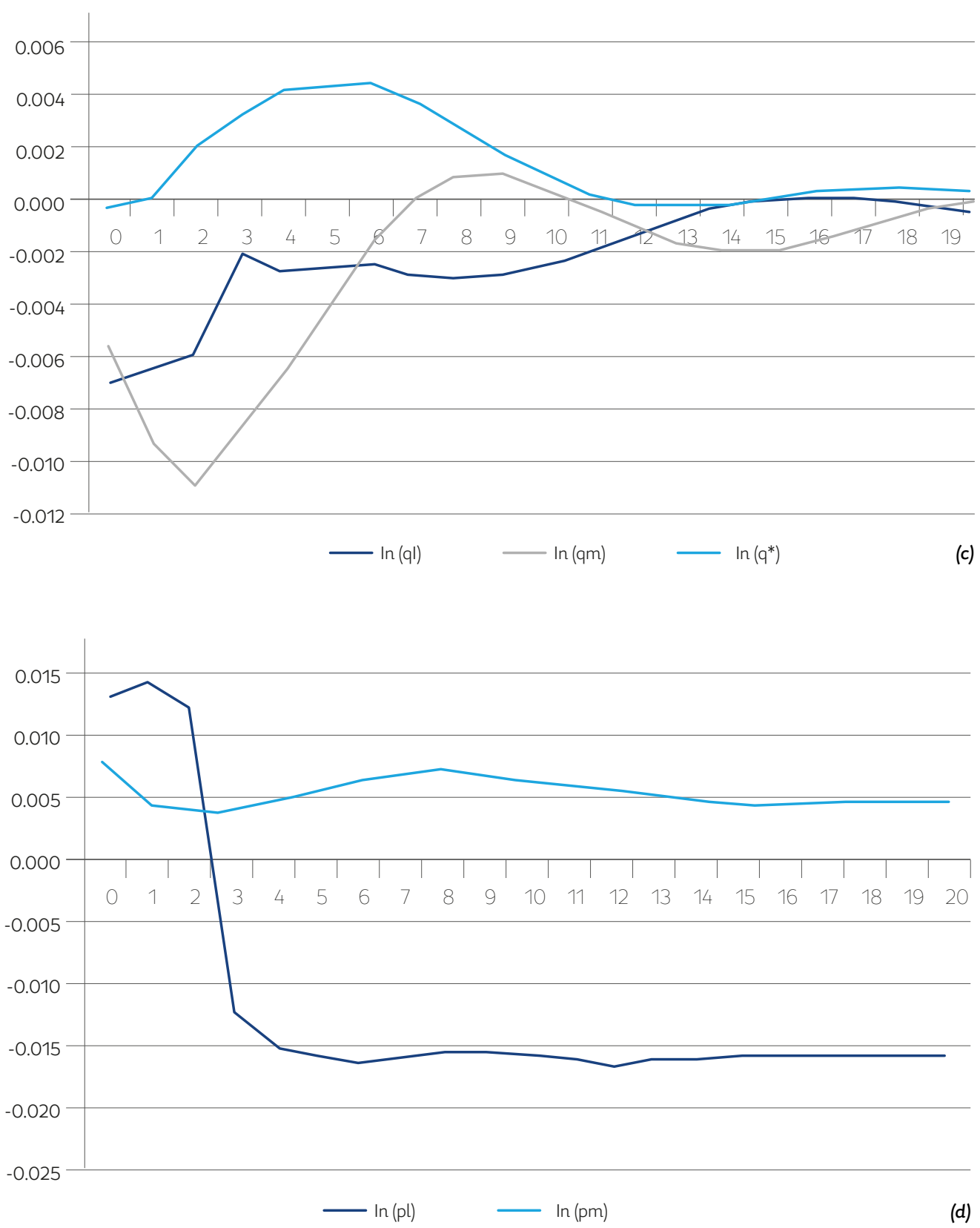
Respuesta del precio minorista
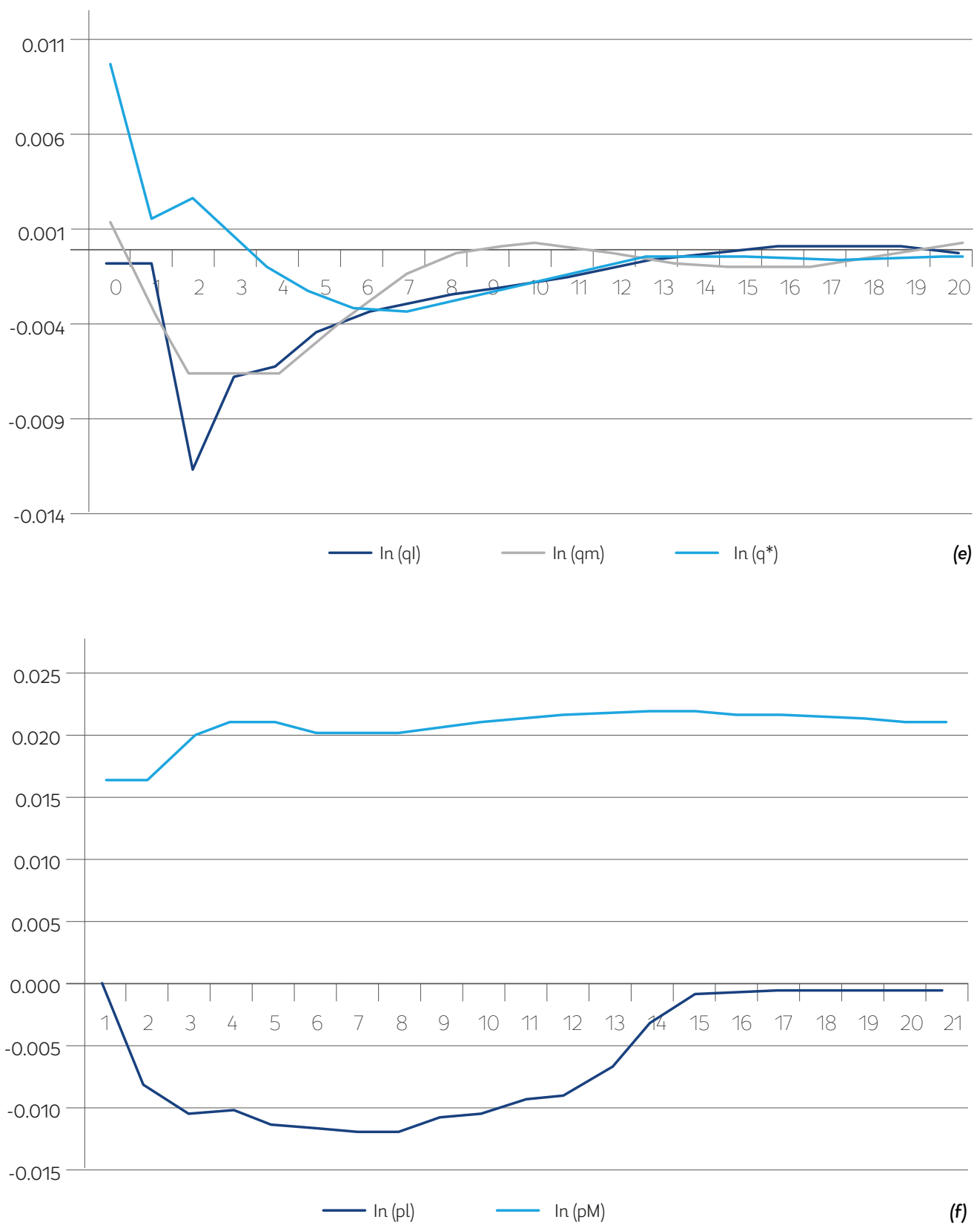

Fuente: elaboración propia

La reacción directa de los precios de leche a cambios imprevistos en las cantidades de lácteos destinadas internamente (aunque de manera temporal) puede deberse a la presión sobre la demanda de leche cruda que afecta los precios, pues este destino de la producción es el más importante en la lechería argentina. Se observa una respuesta directa del precio de la leche cruda a shocks en el resto de los precios con efectos permanentes, lo que muestra la integración vertical de precios (Figura 4.b).

Como se observa en la Figura 4.c., los precios mayoristas reaccionan indirectamente a los shocks en las cantidades de leche cruda y en las de productos destinadas al mercado interno; reacciones esperadas por las posibles presiones de oferta. En cambio, la reacción de los precios mayoristas es directa a variaciones Resulta esperable la transmisión de precios desde el nivel minorista, pero el efecto 
del shock de los precios de la leche cruda no es trivial. Tal efecto puede deberse a que, por ejemplo, un salto positivo no anticipado en el precio de la leche cruda derivaría en mayor oferta de leche cruda, presionando sobre la oferta de productos lácteos y sus precios.

Por otro lado, lo precios minoristas tienen una reacción inversa a los shocks de la cantidad de leche cruda, así como de productos destinados al mercado interno, y ello puede deberse a que la mayor disponibilidad u oferta de lácteos presiona a la baja los precios minoristas. En cambio, integralmente, se observa un efecto directo a shocks en las cantidades de exportación, que puede venir de la escasez relativa en el corto plazo (Figura 4.e).

En general, como resultado, se observa que cambios no previstos en los precios generan efectos permanentes a lo largo de toda la cadena láctea. Un shock positivo en los precios de la leche cruda tiene efectos contrarios sobre las cantidades de lácteos destinadas internamente y sobre los precios mayoristas, pero positivo sobre la cantidad de leche cruda y exportada, con precios minoristas sin cambios a largo plazo. En cambio, shocks positivos de los precios mayoristas y minoristas generan aumentos de las cantidades de leche cruda y de productos lácteos en el mercado interno, con precios superiores en ambos casos, pero las exportaciones caen. Estos resultados son especialmente destacables ya que no se han encontrado antecedentes que aborden la temática de una manera dinámica multinivel de la cadena láctea argentina. 


\section{Conclusiones}

El trabajo se centra en el análisis dinámico de una cadena productiva, teniéndola en cuenta de un modo integral, es decir, incorporando los niveles que la componen. En particular, se aplica al caso de la cadena láctea, una de las actividades económicas más importantes en Argentina, que, aunque ha sido objeto de estudios para comprender su funcionamiento, se critica la incapacidad para analizarla integralmente, con demasiado pragmatismo. Así, el objetivo ha sido analizar las relaciones estables sin perder de vista la integralidad de la cadena, y teniendo en cuenta tanto precios como cantidades de todos sus niveles; además, se incluyeron variables exógenas de oferta y demanda de dichos niveles.

Utilizando una metodología de SVEC, se estudiaron las relaciones de largo plazo entre dichas variables y su dinámica mediante el procedimiento IRA de descomposición de los errores. Entre los resultados más relevantes, se encontró que existen relaciones estables entre cantidades y precios, tanto en el corto como en el largo plazo. En el largo plazo, las cantidades primarias y las que se ubican en el mercado interno están relacionadas de manera positiva con los precios mayoristas y minoristas, pero no se encontró estabilidad con los precios de la leche cruda; en cambio, las exportaciones están relacionadas positivamente con estos últimos, pero negativamente con los primeros.

Estos resultados son de difícil comparación y discusión con los obtenidos en otros estudios, ya que no se han encontrado trabajos con un abordaje similar. Tangencialmente puede mencionarse el trabajo de García Arancibia, Depetris Guiguet, Vicentin Masaro y Rossini (2013), quienes analizaron la decisión de venta de los industriales mayoristas sobre el trade off entre el mercado interno y externo. Teniendo en cuenta solo un nivel de la cadena, han encontrado que los precios, variables coyunturales y productivas son un factor clave en la decisión de venta, conclusión que se comparte en el presente trabajo, lo que afirma que deben ser incorporadas en los análisis dinámicos sectoriales. El de estos autores es uno de los pocos estudios que modela variables productivas con precios de manera endógena, aunque solo tienen en cuenta variables de oferta en un nivel. Con los demás estudios encontrados no se pueden comparar resultados, porque están aplicados a otros mercados con características de funcionamiento distintas a las de Argentina, aunque se coincide en los precios como variables claves de desempeño.

Se considera pertinente proponer ciertas modificaciones para incorporarlas en futuras investigaciones, que pueden complementar este trabajo. En un sentido metodológico, primero, se deben agregar restricciones estructurales sobre el proceso autoregresivo para evaluar si mejoran la capacidad predictiva del modelo. Segundo, y aunque actualmente no resulta posible debido a la escasez de información, se sugiere modelar la variabilidad dentro de cada nivel mediante información desagregada, lo que contribuiría a comprender el funcionamiento sectorial. Tercero, desde un punto de vista más práctico, se debería analizar si las cantidades de exportaciones son estrictamente decisiones de los agentes, ya que en los últimos diez años en Argentina ha habido políticas de restricción sobre ellas, lo que limita las capacidades de gestión. Y, por último, habría que incorporar información de stocks dentro del sistema.

Cabe mencionar que para el presente trabajo se realizó un importante esfuerzo en la obtención de información, ya que en países como Argentina la información disponible tiene la característica de ser deficiente, escasa, o discontinua, lo que complica la realización de análisis formales actualizados. 


\section{Referencias}

Arias, E., \& Torres, C. (2004). Modelos VAR y VECM para el pronóstico de corto plazo de las importaciones de Costa Rica (N.o DIE-01-2004-DI). Costa Rica: Departamentos de Investigaciones Económicas.

Cakir, M., \& Balagtas, I. V. (2012). Estimation market power of U. S. dairy cooperatives in the fluid milk market. American Journal of Agricultural Economics, 94(3), 647-658. https://doi.org/10.1093/ajae/aar136

Davidson, J. E. H., Hendry, D. F., Srba, F., \& Yeo, S. (1978). Econometric modelling of the aggregate time-series relationship between consumers expenditure and income in the United Kingdom. The Economic Journal, 88(352), 661-692.

Depetris Guiguet, E., García Arancibia, R., \& Coronel, M. N. (2013). Impacto del mercado doméstico sobre las exportaciones lácteas santafesinas. Revista de Investigaciones de La Facultad de Ciencias Agrarias, 10(1), 27-32.

Depetris Guiguet, E., Rossini, G., García Arancibia, R., \& Vicentin Masaro, I. (2011). Competitividad del complejo lácteo santafesino. Período 2000-2009. Santa Fe: Facultad de Ciencias Económicas, UNL.

Fernández, S. I. (2007). Estimaciones de demanda de alimentos utilizando un sistema LINQUAD. Recuperado de http://nulan.mdp.edu.ar/622/

Flórez, D. H., Morales, A., Uribe, C. P., \& Contreras, C. A. (2012). Análisis de tendencias en investigación básica para cadenas productivas agroindustriales. Corpoica. Ciencia y Tecnología Agropecuaria, 13(2), 121135.

Gallacher, M. (2000). Cambio tecnológico a nivel desagregado en el agro argentino. En CEMA Working Papers: Serie Documentos de Trabajo (N.o 162). Buenos Aires: Universidad del CEMA.

García Arancibia, R., Depetris Guiguet, E., Vicentin Masaro, I., \& Rossini, G. E. (2013). Factores determinantes de la oferta exportadora láctea santafesina. SaberEs, 1(5), 67-82.

Guerrero, C., \& Lilia, V. (2016). Análisis de alternativas comerciales para la internacionalización de la producción láctea de la Asociación Productora y Comercializadora Agropecuaria Taya del Cantón Tulcán, provincia del Carchi. Universidad Politécnica Estatal de Charchi.

Ibarra Salazar, I., \& García Pérez, F. (2016). Las demandas de factores productivos en la industria maquiladora. Estudios Económicos, 31(2), 265-303.

Johansen, S. (1988). Statistical analysis of cointegration vectors. Journal of Economic Dynamics and Control, 12(2-3), 231-254.

Lajdová, Z., \& Bielik, P. (2013). Vertical price transmission analysis: the case of milk in the SloVak dairy Sector. Applied Studies in Agribusiness and Commerce, 7(1), 89-96.

Lema, D. R. (2006). El crecimiento de la agricultura argentina: un análisis de productividad y ventajas comparativas. Recuperado de https://inta.gob.ar/documentos/el-crecimiento-de-la-agricultura-argentina-un-analisis-de-productividad-y-ventajas-comparativas

Lütkepohl, H. (2005). New introduction to multiple time series analysis. Berlin: Springer.

Lütkepohl, H., \& Krätzig, M. (2005). VECM Analysis in MulTi. Recuperado de http://www.jmulti.de/download/ help/vecm.pdf

Maddala, G. S., \& Kim, I.-M. (1998). Unit roots, cointegration, and structural change (4th ed.). Cambridge, United Kingdom: Cambridge University Press.

McGuire, M. (2012). Forecasting Class III milk prices in a volatile market. Colorado State University.

Mosheim, R. (2012). A quarterly econometric model for short-term forecasting of the U. S. dairy industry (TB1932). U. S. Department of Agriculture, Economic Research Service.

Nicholson, C. F., \& Stephenson, M. W. (2014). Dynamic market impacts of the dairy margin protection program. Journal of Agribusiness, 32(2).

Observatorio de la Cadena Láctea Argentina (OCLA), (2019). Buenos Aires, Argentina. Recuperado en: http:// www.ocla.org.ar/ 
Petrecolla, D. (2016). Estudio sobre las condiciones de competencia en el sector lechero de la República Argentina (informe público).

Pfaff, B. (2008). VAR, SVAR and SVEC models: Implementation within R Package vars. Journal of Statical Software, 27(4), 1-32.

Robledo, W. (2002). Dynamic econometric modeling of the US wheat grain market. Lousiana State University.

Rossini, G., Vicentin Masaro, I., \& Depetris Guiguet, E. (2015). Household cheese consumption in Argentina: A double-hurdle model estimation. 143rd Joint EAAE/AAEA Seminar. Naples, Italy: European Association of Agricultural.

Rossini, G., Vicentin Masaro, J., García Arancibia, R., \& Coronel, M. (2013). Transmisiones de precios en el sector lácteo: el análisis del comportamiento de los precios de exportación y el recibido por los productores. Fave. Sección Ciencias Agrarias, 12(1), 43-52.

Santeramo, F. G. (2014). On the estimation of supply and demand elasticities of agricultural commodites. Recuperado de https://mpra.ub.uni-muenchen.de/56126/

Sexton, R., Sheldon, I., Mccorriston, S., \& Wang, H. (2004). Analyzing vertical market structure and its implications for Agricultural Trade Liberalization. Agricultural Issue Center. University of California, Davis, $1(1), 32$.

Vicentin Masaro, I., Depetris Guiguet, E., García Arancibia, R., \& Rossini, G. (2013). Retrasos en la transmisión de precios de exportación entre los principales productos lácteos santafesinos: leche en polvo entera y quesos. Ciencias Económicas, 2(10), 11-21.

Vicentin Masaro, I., Depetris Guiguet, E., Rossini, G., \& Coronel, M. (2012). Transmisión vertical entre precios de exportación de quesos y precios pagados al productor lechero argentino. XLIII Reunión Anual de La Asociación Argentina de Economía Agraria. Corrientes, Argentina: AAEA.

Wang, Q., Parsons, R., \& Zhang, G. (2010). China's dairy markets: trends, disparities, and implications for trade. China Agricultural Economic Review, 2(3), 356-371.

Yu, X. (2012). Productivity, efficiency and structural problems in Chinese dairy farms. China Agricultural Economic Review, 4(2), 168-175.

Zhang, Y., \& Li, C.-X. (2015). Analysis of the influence of the price factor on the yield of raw milk based on the VEC model. Heilongjiang Animal Science and Veterinary Medicine, 8(1). 


\section{Anexos}

En las tablas A1, A2 y A3 se muestran los resultados de los parámetros estimados de la estructura autorregresiva, las variables exógenas y de las determinísticas, respectivamente.

Tabla A1. Estimadores del VECM correspondientes a la estructura autoregresiva.

\begin{tabular}{|c|c|c|c|c|c|c|c|}
\hline Rezago & $\begin{array}{l}\text { Var. } \\
\text { End. } \\
\text { Rez. }\end{array}$ & $\Delta \ln \left(q_{1}\right)$ & $\Delta \ln \left(q_{m}\right)$ & $\Delta \ln \left(q_{*}\right)$ & $\Delta \ln \left(p_{1}\right)$ & $\Delta \ln \left(\mathbf{P}_{M}\right)$ & $\Delta \ln \left(P_{m}\right)$ \\
\hline \multirow{7}{*}{$t-1$} & $\Delta \ln (q)$ & $0,01[0,878]$ & $-0,229[0,211]$ & $0,12[0,786]$ & $0,018[0,701]$ & $0,02[0,531]$ & $-0,013[0,822]$ \\
\hline & $\Delta \ln \left(q_{m}\right)$ & $-0,055[0,143]$ & $-0,101[0,318]$ & $0,139[0,570]$ & $-0,031[0,235]$ & $-0,021[0,242]$ & $-0,016[0,603]$ \\
\hline & $\Delta \ln \left(q_{*}\right)$ & $-0,029[0,123]$ & $-0,064[0,204]$ & $-0,214+[0,079]$ & $-0,014[0,304]$ & $-0,005[0,562]$ & $0,007[0,644]$ \\
\hline & & & & & & & \\
\hline & $\Delta \ln \left(p_{1}\right)$ & $-0,052[0,643]$ & $0,11[0,718]$ & $-1,289+[0,079]$ & $0,323^{*}[0,000]$ & $-0,04[0,455]$ & $0,139[0,138]$ \\
\hline & $\Delta \ln \left(p_{M}\right)$ & $-0,01[0,950]$ & $-0,148[0,719]$ & $0,814[0,410]$ & $0,159[0,135]$ & $-0,149 * *[0,038]$ & $-0,023^{+}[0,058]$ \\
\hline & $\Delta \ln \left(p_{m}\right)$ & $0,05[0,642]$ & $-0,154[0,597]$ & $1,276^{+}[0,070]$ & $-0,047[0,536]$ & $0,015[0,768]$ & $-0,13[0,148]$ \\
\hline \multirow{7}{*}{$t-2$} & $\Delta \ln \left(q_{1}\right)$ & $0,05[0,476]$ & $-0,168[0,375]$ & $-0,386[0,397]$ & $-0,12 *[0,014]$ & $0,018[0,594]$ & $0,066[0,254]$ \\
\hline & $\Delta \ln \left(q_{m}\right)$ & $-0,072 * *[0,023]$ & $-0,066[0,440]$ & $-0,255[0,219]$ & $-0,026[0,248]$ & $-0,014[0,350]$ & $-0,028[0,289]$ \\
\hline & $\Delta \ln \left(q_{*}\right)$ & $-0,036^{* *}[0,025]$ & $-0,055[0,207]$ & $-0,158[0,134]$ & $-0,008[0,464]$ & $-0,008[0,271]$ & $-0,004[0,755]$ \\
\hline & & & & & & & \\
\hline & $\Delta \ln \left(p_{1}\right)$ & $-0,259 * *[0,024]$ & $0,138[0,657]$ & $-0,937[0,212]$ & $0,196^{* *}[0,016]$ & $0,121^{* *}[0,026]$ & $0,11[0,250]$ \\
\hline & $\Delta \ln \left(P_{M}\right)$ & $0,29^{+}[0,065]$ & $1,239 *[0,004]$ & $1,742^{+}[0,090]$ & $0,016[0,885]$ & $-0,01[0,897]$ & $0,062[0,639]$ \\
\hline & $\Delta \ln \left(p_{m}\right)$ & $-0,026[0,812]$ & $-1,062^{*}[0,000]$ & $-0,64[0,369]$ & $-0,166 * *[0,031]$ & $-0,08[0,124]$ & $-0,011[0,906]$ \\
\hline \multirow{7}{*}{$t-3$} & $\Delta \ln (q)$ & $0,011[0,864]$ & $-0,019[0,910]$ & $-0,329[0,416]$ & $-0,054[0,214]$ & $-0,049 * *[0,095]$ & $0,039[0,455]$ \\
\hline & $\Delta \ln \left(q_{m}\right)$ & $-0,029[0,268]$ & $0,116^{+}[0,099]$ & $0,053[0,756]$ & $0,008[0,662]$ & $0,012[0,315]$ & $-0,015[0,481]$ \\
\hline & $\Delta \ln \left(q_{*}\right)$ & $-0,017[0,155]$ & $-0,017[0,600]$ & $0,084[0,284]$ & $-0,008[0,370]$ & $0,005[0,368]$ & $0,004[0,720]$ \\
\hline & Alnge & 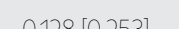 & (2/3 307601 & $0511[0 / 85]$ & $0036[06,5]$ & & \\
\hline & $\Delta \ln \left(p_{1}\right)$ & $-0,128[0,253]$ & $-0,343[0,260]$ & $-0,511[0,485]$ & $0,036[0,645]$ & $-0,01[0,848]$ & $0,148[0,113]$ \\
\hline & $\Delta \ln \left(p_{M}\right)$ & $0,448 *[0,004]$ & $0,576[0,177]$ & $2,315 * *[0,024]$ & $-0,036[0,747]$ & $-0,114[0,125]$ & $\begin{array}{l}-0,273^{* *} \\
{[0,037]}\end{array}$ \\
\hline & $\Delta \ln \left(P_{m}\right)$ & $-0,117[0,266]$ & $0,186[0,515]$ & $-0,544[0,430]$ & $-0,063[0,393]$ & $-0,024[0,633]$ & $-0,069[0,434]$ \\
\hline
\end{tabular}

Notas: la información entre corchetes son los p-valores, $\Delta$ significa variación, Significativo al nivel *1\%, ** $5 \%,{ }^{+10} \%$,

Fuente: elaboración propia. 
Tabla A2. Estimadores del VECM correspondientes a los efectos de las variables exógenas

\begin{tabular}{c|l|l|l|l|l|l}
\multicolumn{1}{c}{ Variable } & \multicolumn{1}{c}{$\Delta \ln \left(q_{1}\right)$} & \multicolumn{1}{c}{$\Delta \ln \left(q_{m}\right)$} & \multicolumn{1}{c}{$\Delta \ln \left(q_{*}\right)$} & \multicolumn{1}{c}{$\Delta \ln \left(p_{1}\right)$} & $\Delta \ln \left(p_{M}\right)$ & $\Delta \ln \left(p_{m}\right)$ \\
\hline EMAE_b04 & $0,001^{* *}[0,011]$ & $0,005^{*}[0,001]$ & $-0,002[0,646]$ & $0,000[0,827]$ & $0,000[0,402]$ & $0,000[0,578]$ \\
\hline IPC_NG & $0,005^{*}[0,015]$ & $-0,006[0,260]$ & $0,006[0,645]$ & $0,000[0,814]$ & $0,000[0,583]$ & $-0,002[0,115]$ \\
\hline IPIM_NG_04 & $-0,004^{*}[0,021]$ & $0,006[0,245]$ & $-0,006[0,639]$ & $0,000[0,819]$ & $0,000[0,595]$ & $0,003[0,104]$ \\
\hline In_IBIF03 & $0,055[0,141]$ & $0,025[0,800]$ & $0,066[0,784]$ & $0,003[0,924]$ & $0,004[0,802]$ & $0,059^{+}[0,057]$ \\
\hline In_pmaiz & $0,026[0,439]$ & $0,015[0,872]$ & $0,011[0,961]$ & $0,037[0,120]$ & $-0,004[0,785]$ & $-0,034[0,222]$ \\
\hline In_Psoja & $0,015[0,674]$ & $-0,062[0,533]$ & $0,188[0,429]$ & $0,000[0,999]$ & $0,008[0,656]$ & $0,034[0,263]$ \\
\hline In_PXSR & $0,048[0,121]$ & $-0,065[0,437]$ & $0,163[0,418]$ & $-0,015[0,498]$ & $-0,002[0,876]$ & $-0,016[0,528]$ \\
\hline In_salario & $-0,049[0,656]$ & $-0,238[0,421]$ & $0,967[0,175]$ & $0,016[0,837]$ & $0,014[0,789]$ & $-0,11[0,227]$ \\
\hline In_salario_ind & $-0,096 *[0,003]$ & $0,079[0,361]$ & $-0,731 *[0,000]$ & $-0,038+[0,090]$ & $-0,013[0,397]$ & $-0,015[0,560]$ \\
\hline
\end{tabular}

Notas: la información entre corchetes son los p-valores, $\Delta$ significa variación, Significativo al nivel *1\%, ${ }^{* *} 5 \%,{ }^{+} 10 \%$,

Fuente: elaboración propia.

Tabla A3. Estimadores del VECM correspondientes a los efectos de las variables determinísticas

\begin{tabular}{l|c|c|c|c|c|c}
\multicolumn{1}{c|}{ Var, Determ } & $\Delta \ln \left(q_{1}\right)$ & $\Delta \ln \left(q_{m}\right)$ & $\Delta \ln \left(q_{*}\right)$ & $\Delta \ln \left(p_{1}\right)$ & $\Delta \ln \left(p_{M}\right)$ & $\Delta \ln \left(p_{m}\right)$ \\
\hline Compensaciones & $-0,024^{*}[0,037]$ & $0,089^{*}[0,004]$ & $-0,02[0,785]$ & $0,003[0,726]$ & $-0,005[0,337]$ & $\begin{array}{l}-0,019^{* *} \\
{[0,049]}\end{array}$ \\
\hline Estac: Feb-Abr & $0,058^{*}[0,000]$ & $0,063[0,152]$ & $0,253^{* *}[0,017]$ & $-0,014[0,215]$ & $-0,021^{*}[0,007]$ & $-0,015[0,275]$ \\
\hline Estac: May-Jul & $-0,079^{*}[0,000]$ & $-0,063^{+}[0,072]$ & $-0,154^{*}[0,071]$ & $-0,015[0,108]$ & $-0,029^{*}[0,000]$ & $-0,008[0,472]$ \\
\hline Estac: Ago-Oct & $0,087^{*}[0,000]$ & $0,086^{*}[0,005]$ & $-0,015[0,844]$ & $-0,02^{* *}[0,011]$ & $-0,004[0,402]$ & $-0,011[0,235]$ \\
\hline Estac, Minorista & $0,103^{*}[0,000]$ & $0,087^{+}[0,058]$ & $0,155[0,159]$ & $0,016[0,191]$ & $-0,011[0,182]$ & $-0,003[0,854]$ \\
\hline Inundaciones y & $-0,041^{*}[0,009]$ & $-0,056[0,190]$ & $-0,172+[0,094]$ & $0,015[0,176]$ & $0,013^{+}[0,090]$ & $0,032^{* *}$ \\
\hline Sequías & $-0,014]$ \\
\hline Retenciones & $-0,042^{*}[0,012]$ & $-0,043[0,344]$ & $-0,068[0,537]$ & $-0,011[0,343]$ & $0,003[0,666]$ & $-0,009[0,517]$ \\
\hline t & $0,000[0,803]$ & $0,001[0,843]$ & $-0,010[0,229]$ & $0,000[0,818]$ & $0,000[0,797]$ & $0,001[0,391]$
\end{tabular}

Notas: la información entre corchetes son los p-valores, $\triangle$ siǵnifica variación, Siǵnificativo al nivel *1\%, ** $5 \%,{ }^{+} 10 \%$,

Fuente: elaboración propia. 